\title{
Quantum System in Contact with a Thermal Environment: Rigorous Treatment of a Simple Model*
}

\author{
P. de Smedt ${ }^{1,2}$, D. Dürr ${ }^{1,3}$, J. L. Lebowitz ${ }^{1,4}$ and C. Liverani ${ }^{1}$ \\ 1 Department of Mathematics, Rutgers University, New Brunswick, NJ 08903, USA \\ 2 Senior Research Assistant of the Belgian National Fund for Scientific Research: New permanent \\ address: Koninklijke Laboratoria Shell, Amsterdam, The Netherlands \\ 3 Heisenberg fellow, Permanent address: Bibos Universität Bielefeld, D-4800 Bielefeld, FRG \\ 4 Department of Physics, Rutgers University, New Brunswick, NJ 08903, USA
}

\begin{abstract}
We study the quantum dynamics of a particle of mass $M$ in an external potential $V(Q)$, interacting with a simple model environment-a harmonic chain of $2 N$ particles with mass $m$ and spring constant $k$. The classical version of this model was studied by Rubin and is equivalent to standard models of a particle interacting with a phonon bath. Setting $m=m^{*} / L$ and $k=k^{*} L$, we prove that for a suitable class of potentials $V$ and initial states $\omega_{0}$, the time evolution of the mass $M$ particle converges, when $N \rightarrow \infty$ and $L \rightarrow \infty$, to the time evolution governed by the Quantum Langevin Equation (QLE) which has been found by Ford, Kac and Mazur. Furthermore we show that, for this class of potentials, the QLE has a unique solution for all positive times, such solution can be expressed as a convergent expansion in the deviation of $V(Q)$ from a harmonic potential. The equilibrium properties of the particle with mass $M$ can be expressed in terms of an integral, over path space, with a Gaussian measure which has mean zero and covariance proportional to $\left[-\Delta+\eta h / M \sqrt{-\Delta}^{-1}\right.$; where $\eta=2 \sqrt{\mathrm{km}}$ is the friction constant, and $h$ is the Plancks' constant (divided by $2 \pi$ ).
\end{abstract}

\section{Introduction}

The behaviour of quantum systems in a dissipative environment is a problem of fundamental and continuing interest [1]. More precisely, one wishes to study the quantum dynamics of a small system (or selected degrees of freedom) in contact with a much larger system - the latter is to be thought of as a heat bath with an essentially infinite number of degrees of freedom. These dynamics are to take account, in a hopefully simple way, of the esssential effects produced by the environment. We shall use the letters $S, E$, and $T$ to denote respectively the system, the environment and their union, i.e. the total (isolated) system.

\footnotetext{
* Supported in part by AFOSR Grant No. 86-0010
} 
Since the system $S$ is not isolated, we expect its dynamics to contain a stochastic element (in contrast to the deterministic evolution of the wave function of the isolated system $T$, given by the Schrödinger equation). The problem is well understood, conceptually at least, for systems evolving according to classical dynamics, where the separation into system and bath degrees of freedom is unambiguous. In particular it is possible to find simple classical examples of a (Brownian) particle, $S$, coupled to a model environment, for which one can rigorously prove that, in a suitable scaling limit, the reduced dynamics are given by the classical Langevin equation (CLE) [2], [4],

$$
\begin{aligned}
M \dot{q}(t) & =p(t), \\
\dot{p}(t)+V_{\text {eff }}^{\prime}(q) & =-(\eta / M) p(t)+F(t) .
\end{aligned}
$$

Here $q(t)$ and $p(t)$ are the position and momentum of the Brownian particle, our system (which for simplicity is taken to be one dimensional); $M$ is the mass, and $V_{\text {eff }}(q)$ is an effective potential acting on the system (consisting of an externally applied potential $V(q)$ and the adiabatic non-dissipative effects of the bath). The right side of (1.1) represents the "dissipative" effects of the environment: the first term is the systematic effect, $\eta$ being the friction coefficient, while the second term $F(t)$ is a fluctuating force; it is Gaussian white noise with

$$
\begin{aligned}
\langle F(t)\rangle & =0, \\
\left\langle F(t) F\left(t^{\prime}\right)\right\rangle & =2(\eta / \beta) \delta\left(t-t^{\prime}\right),
\end{aligned}
$$

where $\beta^{-1}$ is the temperature of the environment.

It is an easily verified fact [3] that there exists a well defined time-homogeneous Markov process with continuous trajectories, the Ornstein-Uhlenbeck (OU) process in an external potential $V_{\text {eff }}(q)$, whose realizations satisfy (1.1). In particular, starting from any initial configuration $q(0)=\bar{q}, p(0)=\bar{p}$ the CLE leads, as $t \rightarrow \infty$, to the stationary single time distribution of the OU process, which has the general form.

$$
\rho_{s}(q, p)=Z^{-1} \exp -\beta\left[p^{2} / 2 M+V_{\text {eff }}(q)\right] .
$$

By requiring $\rho_{s}$ to be the correct marginal distribution for the system at the temperature of the bath, when system + bath are in a Gibbs state with reciprocal temperature $\beta, V_{\text {eff }}(q)$ is identified. (When $\exp \left[-\beta V_{\text {eff }}(q)\right]$ is not integrable, one has to understand (1.2) in a generalized sense.)

While it is clear that (1.1) represents an idealization of the effects of the environment, holding precisely only in an appropriate limit, it is generally accepted that it is, in many cases, a very good approximation to the actual behavior of a massive particle immersed in a fluid. In fact the CLE, or its direct generalizations, have been used as a paradigm for modeling the evolution of a wide variety of open systems (or the dynamics of a reduced set of variables). Its attractiveness lies in its intuitive appeal, mathematical simplicity and in the property that as far as dissipation is concerned all the details of the bath and of the system-bath inter- 
actions are represented by a single parameter, the friction constant $\eta$. This is of course a very welcome simplification which compensates (in most cases) for the loss of some quantitative details due to the idealization [4].

It is clearly desirable to have a quantum version of (1.1), i.e. one that would be useful in situations where quantum effects, such as tunneling, are present. An important step in this direction was taken by Ford, Kac, and Mazur (FKM) in their seminal paper [5]. They considered a particularly simple model, namely a particle coupled linearly to a set of harmonic oscillators. Choosing judiciously the frequency distribution of the latter, they showed that in a certain limit, the classical version of their model satisfied (1.1) with $V_{\text {eff }}(q)$ equal to $V(q)$. In the quantum case they argued that in the same limit the Heisenberg equations of motion for the position and momentum operators will be

$$
\begin{aligned}
M \dot{Q}(t) & =P(t), \\
\dot{P}(t)+(i h)^{-1}[P, V(Q)] & =-(\eta / M) P(t)+W(t),
\end{aligned}
$$

with the $W(t)$ a family of non-commuting Gaussian operators representing "quantum noise" (QN) whose commutators are $c$-numbers,

$$
\left[W(t), W\left(t^{\prime}\right)\right]=\frac{-2 \eta h}{2 \pi} \int_{-\infty}^{\infty} d k k e^{-i k\left(t-t^{\prime}\right)}
$$

and whose expectations are,

$$
\begin{aligned}
\langle W(t)\rangle & =0, \\
\left\langle W(t) W\left(t^{\prime}\right)\right\rangle & =\frac{2 \eta h}{2 \pi} \int_{-\infty}^{\infty} d k \frac{e^{-i k\left(t-t^{\prime}\right)} k}{\exp [\beta h k]-1} .
\end{aligned}
$$

The friction constant $\eta$ is equal to its classical value and $h$ is Planck's constant divided by $2 \pi$.

N.B. - The QLE (1.3) should not be confused with the "quasiclassical Langevin equation" in which $Q, P$ and $W$ are treated as $c$-numbers and the right-hand side of $(1.3 \mathrm{c})$ is set equal to zero, cf. [1g].

In the limit $h \rightarrow 0$, the commutator (1.3c) goes to zero and (1.3e) goes formally over to (1.1d). For fixed $h$ however the $W(t)$ and consequently also the $P(t)$ are much more singular objects than their classical counterparts (compare (1.3e) with (1.1d)). Furthermore the quantum noise is correlated, and thus any solution of (1.3), if it exists, will be non-Markovian. In particular the noise will be correlated with the initial values $Q(0), P(0)$. This is very different from the classical situation, where the Markovian evolution of the reduced dynamics is a consequence of (and implies) the absence, in the scaling limit, of any correlation between the bath noise in the future and the present state of the system; a separation between system and bath which is unnatural, perhaps even impossible, in quantum systems.

To clarify the nature of the difficulties involved let us consider the case in which $V(Q)=\frac{1}{2} K Q^{2}$. Then the formal solution of Eq. (1.3) can be easily computed (cf. Sect. 4); it is given by 


$$
M Q(t)=\varphi_{t}^{\infty}(0) P(0)+\left[-M\left(\varphi_{t}^{\infty}\right)^{\prime}(0)+\eta \varphi_{t}^{\infty}(0)\right] Q(0)+\int_{0}^{\infty} \varphi_{t}(s) W(s) d s
$$

when $\varphi_{t}^{\infty}$ is the solution of the equation

$$
M\left(\varphi_{t}^{\infty}\right)^{\prime \prime}(s)-\eta\left(\varphi_{t}^{\infty}\right)^{\prime}(s)+K \varphi_{t}^{\infty}(s)=M \delta_{t}(s), \quad s \in \mathbb{R} .
$$

We can square $(1.4 \mathrm{a})$ to obtain,

$$
\begin{aligned}
\left\langle Q(t)^{2}\right\rangle= & M^{-2} \varphi_{t}^{\infty}(0)^{2}\left\langle P(0)^{2}\right\rangle+\left[M^{-1} \eta \varphi_{t}^{\infty}(0)-\left(\varphi_{t}^{\infty}\right)^{\prime}(0)\right]^{2}\left\langle Q(0)^{2}\right\rangle \\
& +M^{-2} \int_{0}^{\infty} \int_{0}^{\infty} d s d \tau \varphi_{t}^{\infty}(s) \varphi_{t}^{\infty}(\tau)\langle W(s) W(\tau)\rangle+\text { cross terms. }
\end{aligned}
$$

It is easy to check, using (1.3e), that the third term of (1.5) is infinite for all $t>0$. This shows that if we want $\left\langle Q(t)^{2}\right\rangle$ to be finite, we cannot assume that the $Q(0)$, $P(0)$ are uncorrelated with the noise.

Given the existence of such correlations we clearly need a more complete prescription of the QLE than is provided by (1.3). More precisely we need to address the following questions:

i) For which initial states $\omega_{0}$, i.e. density matrices, of $T$ is a derivation of the QLE, formally described in (1.3), valid? In particular what kind of correlations between $Q(0), P(0)$ and the noise are induced by these states?

ii) What is the nature of the process described by the solutions of the QLE? E.g. what are the stationary states $\tilde{\omega}_{s}$ for this stochastic process? In particular does the Gibbs state $\omega_{\text {eq }}$ for $T$ lead to a stationary state $\tilde{\omega}_{s}$ for the QLE? One certainly expects this to be so with the projection $\rho_{\mathrm{eq}}=\operatorname{tr}_{\mathrm{E}}\left(\omega_{\mathrm{eq}}\right)$ of $\omega_{\mathrm{eq}}$ on $S$ to yield, in the scaling limit, the single time marginal distribution $\rho_{s}$ of $\tilde{\omega}_{s}$. (The meaning of $\tilde{\omega}_{s}$ here is analogous to the stationary measure on path space for the CLE.)

Assuming the answer to (ii) is positive, there still remains the important question:

iii) What other initial states $\tilde{\omega}$ for the QLE, if any, evolve, as $t \rightarrow \infty$, to $\tilde{\omega}_{s}$ ?

These questions were already raised by FKM and studied further in refs [5-8], (ii) is essentially resolved in the case when $V(Q)$ is a harmonic potential, i.e. $V(Q)=$ $\frac{1}{2} K Q^{2}$. In this case the derivation of (1.3) as done by FKM is valid when the initial state of $T$ is that of equilibrium, i.e. $\omega_{\text {eq }}$. It is then clear that $\omega_{\text {eq }}$ leads to the unique stationary state $\tilde{\omega}_{s}$ for the process described by the QLE. Properties of $\tilde{\omega}_{s}$ can be determined directly from (1.3) or by studying $\omega_{\mathrm{eq}}$ in the proper limit. This is explicitly calculable since $\omega_{\text {eq }}$ is the equilibrium state of a harmonic system. One finds the expected correlation between $P(0)$ and the $W(t)$ and that the removal of the frequency cutoff leads to an infinite value for the expectation of the kinetic energy $\tilde{\omega}_{s}\left(P^{2}\right)$. This is due to the zero point fluctuation of the environment which reflects itself also in the singular noise. Despite this the position process is well defined (this is not the case if the initial state is taken to be a product state). One may also consider the special harmonic case when $K=0$. In this case, there is no well-defined equilibrium state for the system, but one can easily show that $\omega_{s}\left(\left(Q(t)-Q(0)^{2}\right)\right) / t \rightarrow D$ as $t \rightarrow \infty$, where $D=2 / \eta \beta$ is the classical diffusion constant. 
Question iii) too can be answered for the harmonic case, see Sect. 6. In addition it was shown by Maasen [7] that by considering the process described by (1.3) as holding for all $t \in \mathbb{R}$, i.e. no initial conditions, one can construct a stationary measure $\tilde{\omega}_{s}$. He shows further that states $\tilde{\omega}$, which are perturbations of the equilibrium state, induced by modifying the noise over some time interval $(-\tau, 0)$, evolve, when $t \rightarrow \infty$, to the equilibrium state $\tilde{\omega}_{s}$.

As indicated earlier, however, we are primarily interested in the answer to the above questions in the physical situation where the total system $T$ is prepared, or known to be, in a specified state at some initial time, $t=0$. This is an open problem particularly for the anharmonic case which we partially answer in this paper.

To do this we investigate in detail a more concrete model system than that considered by FKM. This model, also studied by Rubin [9], consists of an infinite harmonic chain with nearest neighbor interactions in which the special particle is acted on by an external potential $V(Q)$. Rubin's model can be transformed into the "standard" FKM model where a special degree of freedom is linearly coupled to an independent phonon bath (see e.g. [6]). To obtain dissipation of the Langevin type (linear friction without memory) in the FKM model, one removes the curoff frequency to infinity. This corresponds here precisely to taking the "scaling limit." It is then that the noise spectrum becomes that of the black body radiation (1.3a).

In the case where $V(Q)$ is harmonic the analysis can be done through quasi-free states, where one need only consider convergence of the two point functions in order to get the convergence of the state on bounded "continuous" cylinder functions of the position, which corresponds to the convergence of finite dimensional distributions. We cannot hope to obtain this type of convergence for the nonlinear system through direct computation; this forces us to follow a less direct path. First we construct a class of "good" initial (quasi free) states $\omega$ on $T$ in which the particle is localized at $t=0$. (N.B. the "good" initial states do not include simple product states, which are frequently used in computations.) We then show that, in the scaling limit, the QLE describes the evolution of $Q(t)$ for all $t>0$. This is done by proving that $\exp (i \lambda Q(t))$ has a convergent expansion in terms of both the noise operators and the non-quadratic part of the potential whose properties are known. Finally we prove that the initial states $\omega$ for $T$ lead to states $\tilde{\omega}$ associated with the QLE; to do this we have to develop some machinery in order to rephrase in an algebraic language standard results of classical probability theory (e.g. the Kolmogorov reconstruction theorem).

It should be noted that, since the QLE does not describe a Markov process on the state (Hilbert) space of $S$, explicit computations with the QLE are quite difficult when $V(Q)$ is not harmonic. Thus we don't ask, at this time, about the approach to equilibrium or address more refined questions. (In fact we don't even prove that $\omega_{\text {eq }}$ leads to a good initial state for anharmonic potentials.) These problems are however crucial for making the QLE useful in practice, and are the ultimate motivation for studying it. We hope to use the expansion mentioned above to say something about the time evolution of the system in a double well.

The rest of the paper is organized in the following way: In Sect. 2 we describe the model. The reduced equilibrium behavior of the system is studied in Sect. 3. 
We find there a compact form for the reduced density matrix $\rho_{\text {eq }}$ in the scaling limit, cf. Bray and Moore [10]. In Sect. 4, we describe the reduced dynamics and state the convergence result, which is proven in Sect. 5. Comments about the solution of the QLE for the harmonic case and related open problems are given in Sect. 6. Finally, the appendices (and Sect. 5) contain most of the technical work: the other parts can be read independently.

\section{The Model}

We take as our total system $T$ a 1 -dimensional harmonic chain containing $2 N+1$ particles with periodic boundary conditions: $2 N$ of these particles have mass $m$ and will be regarded as the environment, $E$, (bath) which interacts with a particle of mass $M>m$, the system $S$. The Hamiltonian of the total system is

where

$$
H^{V}=H_{0}+V\left(Q_{0}\right)
$$

$$
H_{0}=\frac{1}{2 m} \sum_{\substack{j=-N \\ j \neq 0}}^{N} P_{j}^{2}+\frac{1}{2 M} P_{0}^{2}+\frac{k}{2} \sum_{j=-N}^{N}\left(Q_{j+1}-Q_{j}\right)^{2},
$$

$Q_{N+1} \equiv Q_{-N}$, and $V\left(Q_{0}\right)$ is an external potential felt by the zeroth particle.

The classical version of this model with $V=0$ was studied by Rubin [9]. He showed that when the environment was in equilibrium at a temperature $\beta^{-1}$ then in the limit $N \rightarrow \infty$ the heavy particle diffuses with diffusion constant $2 / \beta \sqrt{\mathrm{km}}$. He showed moreover that, in the limit $M \gg m$, the heavy particle obeys, for times scaled by $\sqrt{M}$, the classical Langevin equation with $V_{\text {eff }}=0$. Rubin's work has been generalized and made rigorous by various authors [11].

Repeating Rubin's analysis in the quantum case one sees that for large $M$ quantum effects become unimportant and one obtains the CLE. We therefore consider a different scaling which is equivalent to the previous scaling in the classical case, but retains the quantum mechanical properties of the system. Parameterizing $m$ and $k$ by $m=m^{*} / L$ and $k=k^{*} L$ we consider the thermodynamic limit $N \rightarrow \infty$ followed by the scaling limit $L \rightarrow \infty$. (We shall sometimes denote by "scaling limit" both of these limits.)

For technical reasons, we will restrict ourselves to the class of potentials consisting of a quadratic part plus a bounded part $\tilde{V}$,

$$
V(x)=\frac{1}{2} K x^{2}+\int_{\mathbb{R}} e^{i \lambda x} d v(\lambda), \quad x \in \mathbb{R},
$$

where $v(s)$ has even real and odd imaginary parts, and

$$
\int_{\mathbb{R}} \lambda^{2}|d v(\lambda)|<\infty .
$$

The Hamiltonian $H^{V}$ can be defined as a self-adjoint operator on $L^{2}\left(\mathbb{R}^{2 N+1}\right)$. As shown in Appendix 1, it can be transformed via a canonical transformation into a diagonal form for the bath variables,

$$
H^{V}=\frac{1}{2 m} \sum_{j=1}^{2 N} p_{j}^{2}+\frac{1}{2 M} P^{2}+\frac{1}{2} \sum_{j=1}^{2 N} \lambda_{j}^{2}\left(q_{j}-\delta_{j} Q\right)^{2}+V(Q) .
$$


Here $Q$ and $P$ stand for $Q_{0}$ and $P_{0}, \lambda_{j}$ is the eigenfrequency of the $j^{\text {th }}$ normal mode $\left(q_{j}, p_{j}\right)$, and $C_{j}=-\lambda_{j}^{2} \delta_{j}$ is the coupling of our system to the $j^{\text {th }}$ mode. We note that Hamiltonians of the form (2.5) are widely used in the literature on Quantum Brownian motion, see e.g. $[1,4-8,12-15]$. The main difference here is that the frequencies $\lambda_{i}$ and couplings $\delta_{i}$ are fully determined by $k$ and $m$. In particular our model corresponds, in the thermodynamic limit, to the "ohmic" dissipative model studied in [6], i.e. in the limit $N \rightarrow \infty$ our "density of states" becomes $\rho(\lambda)=$ $2\left(4 k / m-\lambda^{2}\right)^{-1 / 2}$ and the couplings $C_{j}$ become $C(\lambda)$, where

$$
C(\lambda)^{2} \rho(\lambda) \cong\left\{\begin{array}{lll}
\frac{2 m}{\pi} \lambda^{2} & \text { if } & |\lambda| \leqq 2 \sqrt{k / m} \\
0 & \text { if } & |\lambda|>2 \sqrt{k / m}
\end{array}\right.
$$

Next we consider the initial condition, i.e. the state of the total system at time 0 . For classical systems this is easy: to obtain the CLE for the most general initial state of $S$ we specify $Q(0)$ and $P(0)$ and take for the bath the thermal equilibrium state of the whole system $T$ conditioned on the initial momentum and position of the zero particle. It is not clear how to translate this type of initial state to quantum mechanics since $Q$ and $P$ cannot be specified simultaneously and the conditioning is not well defined. A common choice is to start with a product state for system and bath, i.e. the particle is effectively decoupled from the bath at time 0 . As we have, however, already noted in the introduction and shall show in more detail later, this leads to singular dynamics in the scaling limit: we believe this problem to be intrinsic to all models of the QLE.

In the search for suitable initial states an essential desiderata is to localize the zero particle at the starting time. In order to do so we consider initial states $\omega_{K_{0}}$ of the form

$$
\omega_{K_{0}}=e^{-\beta H^{V_{0}}} / \operatorname{Tr} e^{-\beta H^{V_{0}}}
$$

where $V_{0}(x)=\frac{1}{2} K_{0} x^{2}$. Note that $K_{0}$ need not equal $K$ in (2.3). By adjusting the value of $K_{0}$, we can localize the heavy particle near the origin. The big advantage of these states $\omega_{K_{0}}$ is that they are quasifree (see e.g. [16]), implying that higher order correlations can be easily calculated as soon as the one- and two-point correlation functions are known.

\section{The Reduced Equilibrium State in the Scaling Limit}

Before turning to the dynamics we consider the reduced density matrix $\rho_{\text {eq }}$ of the zeroth particle, $S$, when $T$ is in the equilibrium state $\omega_{\mathrm{eq}}^{N, L}$ at termperature $\beta^{-1}$ with respect to the Hamiltonian. (We make here explicit the $N$ and $L$ dependence unless it is completely clear from the context.) As mentioned in the introduction, it seems extremely plausible that the restriction of this state to $S$ agrees, in the scaling limit, with the stationary state of the QLE, restricted to functions of the position at a single time. Our results are consistent with the (properly interpreted) expressions of Bray and Moore [10], We also find a compact formula for the imaginary time correlation functions of the particle in the $\lim \lim \omega_{\text {eq }}^{N, L}$. 
The reduced equilibrium density matrix $\rho_{\mathrm{eq}}^{N, L}(x, y)$ of the zeroth particle is given by,

$$
\rho_{\mathrm{eq}}^{N, L}(x, y)=\frac{\int d x_{1} \ldots d x_{2 N} \exp -\beta H_{N, L}^{V}\left(x, x_{1}, \ldots x_{2 N} ; y, x_{1}, \ldots x_{2 N}\right)}{\int d x_{0} d x_{1} d x_{2 N} \exp -\beta H_{N, L}^{V}\left(x_{0}, x_{1} \ldots x_{2 N} ; x_{0}, x_{1} \ldots x_{2 N}\right)} .
$$

Given $\rho_{\text {eq }}^{N, L}$ we can calculate the expectation values of the position and momentum observable of the zeroth particle in this Gibbs state $\omega_{\mathrm{eq}}^{N, L}$, since:

$$
\begin{aligned}
& \omega_{\mathrm{eq}}^{N, L}(\exp i \lambda Q)=\int d x \rho_{\mathrm{eq}}^{N, L}(x, x) \exp i \lambda x, \\
& \omega_{\mathrm{eq}}^{N, L}(\exp i \mu P)=\int d x \rho_{\mathrm{eq}}^{N, L}(x, x+\mu h) .
\end{aligned}
$$

We use the Feynman-Kac formula to write down an expression for the kernel $\exp -\beta H_{N, L}^{V}\left(x, x_{1}, \ldots x_{2 N} ; y, y_{1}, \ldots, y_{2 N}\right)$. As usual, since $H^{V}$ is quadratic in the bath variables we may integrate these out explicitly and get,

$$
\begin{aligned}
\rho_{\mathrm{eq}}^{N, L}(x, y)= & C_{N, L} \exp -\frac{h^{2}(x-y)^{2}}{2 M \beta} \\
& \cdot E^{w}\left\{\exp -\left(\int_{0}^{\beta} V(\omega(s)+x) d s+I_{N, L}(\omega)\right) \mid \omega(\beta)=y-x\right\} .
\end{aligned}
$$

In this expression $E^{w}$ denotes the expectation value with respect to the Wiener process with mean 0 and covariance $E^{w}\{\omega(s) \omega(t)\}=\left(h^{2} / M\right) \min \{s, t\} ; E^{w}\{\cdot \mid \omega(\beta)=$ $y-x\}$ denotes the conditioning that the process passes through the point $y-x$ at time $\beta ; C_{N, L}$ is the normalization constant so that $\int d x \rho_{\mathrm{eq}}^{N, L}(x, x)=1$ and $I_{N . L}(\omega)$ is the influence functional defined by

$$
I_{N, L}(\omega)=\frac{1}{2} \int_{0}^{\beta} d s \int_{0}^{\beta} d t A_{N, L}(s-t)[\omega(s)-\omega(t)]^{2}
$$

where

$$
A_{N, L}(u)=\frac{h}{4 \sqrt{m}} \sum_{j} \lambda_{j}^{3} \delta_{j}^{2} \frac{\cosh \left(\left(\frac{\beta}{2}-|u|\right) h \lambda_{j} / \sqrt{m}\right)}{\sinh \left(\beta h \lambda_{j} / 2 \sqrt{m}\right)} \quad-\beta \leqq u \leqq \beta .
$$

Using the results of Appendix I, (3.4b) can also be written as an integral over the contour $\hat{\Gamma}$ :

$$
A_{N, L}(u)=\frac{k h}{4 \pi i \sqrt{m}} \int_{\hat{\Gamma}} d \xi \frac{1}{G\left(\xi^{2}\right)} \frac{\cosh \left(\left(\frac{\beta}{2}-|u|\right) h \xi / \sqrt{m}\right)}{\sinh (\beta h \xi / 2 \sqrt{m})},
$$

where $G$ is defined in Lemma I.2.

The limit $N \rightarrow \infty$ can be easily taken by applying the Lebesgue dominated convergence theorem. Note that

$$
A_{L}(u) \equiv \lim _{N \rightarrow \infty} A_{N, L}(u)=\frac{h \eta}{4 \pi} \int_{-a}^{+a} d x x \sqrt{1-(x / a)^{2}} \frac{\cosh \left\{\left(\frac{\beta}{2}-|u|\right) h x\right\}}{\sinh \frac{\beta}{2} h x}
$$


where $a=2 L \sqrt{k^{*} / m^{*}}$ and $\eta=2 \sqrt{k^{*} m^{*}}$. In the limit $L \rightarrow \infty, A_{L}$ becomes singular $\left(A_{L}(t) \sim t^{-2}\right)$ but $|\omega(t)|^{2} \sim t$ almost surely for $t \rightarrow 0$ [17], hence the limit needs to be taken with some care. We consider explicitly the case $x=y$ (similar analysis can be done for $x \neq y$ ). The right-hand side of (3.3) may be written as

$$
\tilde{C}_{L} E_{L}\left\{\exp \left[-\int_{0}^{\beta} V(\omega(s)+x) d s\right]\right\}
$$

where we have introduced the Gaussian measures, defined on $C_{0}^{0}([0, \beta])$, (all continuous functions which vanish at the end points) by

$$
E_{L}(\cdot)=\left[E^{w}\left\{\exp -I_{L}(\omega) \mid \omega(\beta)=0\right\}\right]^{-1} E^{W}\left\{\cdot \exp -I_{L}(\omega) \mid \omega(\beta)=0\right\}
$$

with $I_{L}(\omega)=\lim _{N \rightarrow \infty} I_{N, L}(\omega)$.

If we define $a_{k}(f)=\frac{1}{\beta} \int_{0}^{\beta} f(t) \cos \frac{2 \pi k t}{\beta} d t ; b_{k}(f)=\frac{1}{\beta} \int_{0}^{\beta} f(t) \sin \frac{2 \pi k t}{\beta} d t$, then we can represent every function $f \in C_{0}^{0}([0, \beta])$ by the Fourier series $f(t)=$ $\sum_{k=1}^{\infty} a_{k}(f)\left(\cos \frac{2 \pi k t}{\beta}-1\right)+b_{k}(f) \sin \frac{2 \pi k t}{\beta}$, where the convergence holds in many different senses, three of which are relevant here: i) in $L^{2}([0, \beta])$; ii) in $L^{2}\left(C_{0}^{0}([0, \beta]), E^{w}\right)$; iii) pointwise in $t$ almost surely with respect to $E^{w}$. The first sense of convergence implies that $I_{L}(\omega)=\frac{\beta^{2}}{2} \sum_{k=1}^{\infty}\left(a_{k}(\omega)^{2}+b_{k}(\omega)^{2}\right)\left(A_{k, L}-A_{0, L}\right)$, where, using explicitly the symmetries of $A_{L}, A_{L}(t)=\sum_{k=0}^{\infty} A_{k, L} \cos \frac{2 \pi k t}{\beta}$.

Now, an explicit computation yields,

$$
\begin{aligned}
& \lim _{L \rightarrow \infty} E_{L}\left(a_{k} a_{j}\right)=2 \delta_{k j}\left[\frac{M \pi^{2} k^{2}}{\beta h^{2}}+\eta \frac{\pi k}{h^{2} \beta}\right]^{-1}, \\
& \lim _{L \rightarrow \infty} E_{L}\left(a_{k} b_{j}\right)=0, \\
& \lim _{L \rightarrow \infty} E_{L}\left(b_{k} b_{j}\right)=2 \delta_{k j}\left[\frac{M \pi^{2} k^{2}}{\beta h^{2}}+\eta \frac{\pi k}{\beta h^{2}}\right] .
\end{aligned}
$$

This allows us to compute $\lim _{L \rightarrow \infty} E_{L}(\omega(s) \omega(t))$, once we take advantage of the other two senses in which Fourier series converge.

The final results of these computations is,

$$
\begin{aligned}
& \lim _{L \rightarrow \infty} \lim _{N \rightarrow \infty} \rho_{\mathrm{eq}}^{N, L}(x, y)=0 \quad \text { if } \quad x \neq y \\
& \lim _{L \rightarrow \infty} \lim _{N \rightarrow \infty} \omega_{\mathrm{eq}}^{N, L}(\exp i \lambda P)=\delta_{\lambda 0} \\
& \lim _{L \rightarrow \infty} \lim _{N \rightarrow \infty} \omega_{\mathrm{eq}}^{N, L}(\exp i \lambda Q)=\int d x \rho_{\mathrm{eq}}(x) \exp i \lambda x .
\end{aligned}
$$

where 


$$
\rho_{\mathrm{eq}}(x) \equiv \lim _{L \rightarrow \infty} \lim _{N \rightarrow \infty} \rho_{\mathrm{eq}}^{N, L}(x, x)=\frac{E^{\mathscr{G}}\left\{\exp -\int_{0}^{\beta} V(\omega(s)+x) d s\right\}}{\int d x_{0} E^{\mathscr{G}}\left\{\exp -\int_{0}^{\beta} V\left(\omega(s)+x_{0}\right) d s\right\}}
$$

Here $E^{\mathscr{G}}$ denotes the Gaussian process having mean zero and covariance $\left(-\frac{M}{h^{2}} \Delta_{D}+\frac{\eta}{h} \sqrt{-\Delta_{D}}\right)^{-1}$, where $\Delta_{D}$ is the Laplacian with Dirichlet boundary conditions at 0 and $\beta$. To be more explicit, $E^{\mathscr{G}}$ is the Gaussian process defined by

$$
\begin{aligned}
E^{\mathscr{G}}\{\omega(s)\} & =0, \\
E^{\mathscr{G}}\{\omega(s) \omega(t)\} & =f(s)+f(t)-f(s-t) \quad \forall s, t: 0 \leqq s, t \leqq \beta,
\end{aligned}
$$

where

$$
f(s)=\beta \sum_{k=1}^{\infty}\left(1-\cos \frac{2 \pi k s}{\beta}\right)\left[\frac{2 \pi^{2} k^{2} M}{h^{2} \beta^{2}}+\frac{\pi k \eta}{h \beta}\right]^{-1} .
$$

It follows from $(3.8 \mathrm{~b})$ that the limiting state $\omega_{\mathrm{eq}}$ is no longer normal with respect to the algebra of operators generated by $\{\exp i \lambda Q$, $\exp i \mu P\}$, i.e. it is not of Trace class type: see [16]. This implies in particular that, $\lim \lim \omega_{\mathrm{eq}}^{N, L}\left(P^{2}\right)=\infty$.

We can also obtain dynamical correlation functions such as $F(t-s) \equiv$ $\lim _{L \rightarrow \infty} \lim _{N \rightarrow \infty} \omega_{\text {eq }}^{N, L}\left(Q(t)^{n} Q(s)^{m}\right)$. The standard way to obtain $F(u)$ is to look at the imaginary-time correlation function $H(t)$

$$
H_{n, m}(t)=\lim _{L \rightarrow \infty} \lim _{N \rightarrow \infty} \omega_{\mathrm{eq}}^{N, L}\left(Q^{n} Q(i t h)^{m}\right), \quad(0 \leqq t \leqq \beta),
$$

where $Q(i t h)=\exp \left(-t H_{N, L}^{V}\right) \quad Q \exp \left(t H_{N, L}^{V}\right)$. The real-time correlation function $F(u)$ is then found from $H(t)$ by analytic continuation. Similar computations as before yield:

$$
H_{n, m}(t)=\frac{\int d x \int d y x^{n} y^{m} K(x, y ; t)}{\int d x \int d y K(x, y ; t)}
$$

where

$$
K_{x y}(t)=E^{\mathscr{G}}\left\{\delta(\omega(t)+x-y) \exp -\int_{0}^{\beta} V(\omega(s)+x) d s\right\}
$$

\section{The Quantum Langevin Equation-Statement of Results}

The Reduced Dynamics. We consider now the motion of the zeroth particle when the system evolves according to the Hamiltonian $H^{V}$. The Hamiltonian (2.5) determines the dynamics $\tau_{t}$ of the system: $\tau_{t}(A)=\exp \left(i t H^{V} / h\right) A \exp \left(-i t H^{V} / h\right)$. Let us denote the domain of an operator $A$ by $D(A)$. Because $D\left(q_{i}\right)$ and $D\left(p_{i}\right) \supset D\left(H^{V}\right) \supset$ $S\left(\mathbb{R}^{2 N+1}\right)$, we can define $\tau_{t}\left(q_{i}\right)$ and $\tau_{t}\left(p_{i}\right)$; they are essentially self-adjoint on $D\left(H^{V}\right)$. It is then easy to show that for each $\psi \in D\left(H^{V}\right)$, the time evolution of the Heisenberg operators in the state $\psi,\left(\psi, \tau_{t}(A) \psi\right)$, satisfy the equations of motion, 


$$
\begin{aligned}
& \frac{d}{d t}\left(\psi, \tau_{t}\left(q_{i}\right) \psi\right)=m^{-1}\left(\psi, \tau_{t}\left(p_{i}\right) \psi\right), \\
& \frac{d}{d t}\left(\psi, \tau_{t}(Q) \psi\right)=M^{-1}\left(\psi, \tau_{t}(P) \psi\right), \\
& \frac{d}{d t}\left(\psi, \tau_{t}\left(p_{i}\right) \psi\right)=-\lambda_{i}^{2}\left(\psi, \tau_{t}\left[q_{i}-\delta_{i} Q\right] \psi\right), \\
& \frac{d}{d t}\left(\psi, \tau_{t}(P) \psi\right)=\sum_{j=1}^{2 N} \lambda_{j}^{2} \delta_{j}\left(\psi, \tau_{t}\left[q_{j}-\delta_{j} Q\right] \psi\right)-\left(\psi, \tau_{t}\left(V^{\prime}(Q)\right) \psi\right) .
\end{aligned}
$$

Defining the self-adjoint operator $W(t)$ and function $\gamma(t)$ as,

$$
\begin{aligned}
W(t) & =\sum_{j} \lambda_{j}^{2} \delta_{j}\left[\left(q_{j}-\delta_{j} Q\right) \cos \left(\lambda_{j} t / \sqrt{m}\right)+\frac{1}{\sqrt{m}} p_{j} \lambda_{j}^{-1} \sin \left(\lambda_{j} t / \sqrt{m}\right)\right], \\
\gamma(t) & =\sum_{j} \lambda_{j}^{2} \delta_{j}^{2} \cos \left(\lambda_{j} t / \sqrt{m}\right),
\end{aligned}
$$

we can express the time evolution of $S$ in a simple compact form. (Note that everything should really have subscripts $N, L$.)

Lemma 4.1. For each $\psi \in D\left(H^{V}\right), t \in \mathbb{R}^{+}$,

$$
M \frac{d^{2}}{d t^{2}}\left(\psi, \tau_{t}(Q) \psi\right)=-\int_{0}^{t} \gamma(t-s)\left[\frac{d}{d s}\left(\psi, \tau_{s}(Q) \psi\right)\right] d s-\left(\psi, \tau_{t}\left(V^{\prime}(Q)\right) \psi\right)+(\psi, W(t) \psi)
$$

Proof. In (4.1c) we consider $\left(\psi, \tau_{t}(Q) \psi\right)$ to be given unknown function of $t$. Solving (4.1a) and (4.1c), we then get that

$$
\begin{aligned}
\left(\psi, \tau_{t}\left(q_{i}\right) \psi\right)= & \left(\psi, q_{i} \psi\right) \cos \left(\lambda_{j} t / \sqrt{m}\right)+\frac{1}{\sqrt{m}}\left(\psi, p_{i} \psi\right) \lambda_{i}^{-1} \sin \left(\lambda_{j} t \sqrt{m}\right) \\
& +\frac{1}{\sqrt{m}} \int_{0}^{t} \lambda_{i} \delta_{i} \sin \left(\lambda_{i}(t-s) / \sqrt{m}\right)\left(\psi, \tau_{s}(Q) \psi\right) d s .
\end{aligned}
$$

The result is obtained by substituting this expression in (4.1d).

Now, let $\mathscr{B}(J)$ (respectively $\mathscr{B}_{0}(J)$ ) be the completion of the real space $C^{1}(J)$ (respectively $C_{0}^{1}(J)$ ) with respect to the norm $\|f\|=\left|f^{\prime}(0)\right|+\|f\|_{1}+\left\|f^{\prime}\right\|_{1}$, where $\|g\|_{1}=\int_{J}|g(x)| d x$ and $J \subset \mathbb{R}^{n}$ is a closed set. For each $f$ in $\mathscr{B}\left(\mathbb{R}^{+}\right)$, we then define the self-adjoint operator:

$$
B(f)=f(0) M^{-1} P-\left\{f^{\prime}(0)-M^{-1} \int_{0}^{\infty} f(t) \gamma(t) d t\right\} Q+M^{-1} \int_{0}^{\infty} W(t) f(t) d t .
$$

The reduced dynamics of our problem can then be written according to:

Lemma 4.2. The operator $\tau_{t}\left(e^{i \mu Q}\right)\left(\mu \in \mathbb{R} ; t \in \mathbb{R}^{+}\right)$is the unique solution of the equation 


$$
\begin{aligned}
& X(\mu, t)=\exp \left\{i \mu\left[B\left(\varphi_{t}\right)-i M^{-1} \int_{0}^{t}\left[\varphi_{t}(s) \int_{\mathbb{B}} \lambda X(\lambda, s) d \nu(\lambda)\right] d s\right]\right\}, \\
& X(\mu, t)^{*}=X(-\mu, t),
\end{aligned}
$$

where $v(\lambda)$ is defined in (2.3), the integral is in the strong sense (Bochner) and $\varphi_{t}(s)=\phi(s-t) . \phi(s)$ is the solution of the equation

$$
\begin{aligned}
& M \phi^{\prime \prime}(s)-\int_{s}^{0} d \tau \gamma(\tau-s) \phi^{\prime}(\tau)+K \phi(s)=0, \quad s<0, \\
& \phi(0)=0, \quad \phi^{\prime}(0)=-1, \quad \text { and } \phi(s)=0 \quad \text { for } \quad s>0 .
\end{aligned}
$$

Proof. We start by multiplying both sides of Eq. (4.3) by a function $f \in \mathscr{B}\left(\mathbb{R}^{+}\right)$with $f(0)=0$ and $f^{\prime}(0)=-1$. After some integration by parts, we get:

$$
\begin{aligned}
\int_{0}^{t}\left(\psi, \tau_{s}(Q) \psi\right)\left[M f^{\prime \prime}(s)-\int_{s}^{t} d \tau \gamma(\tau-s) f^{\prime}(\tau)+K f(s)\right] d s+M\left(\psi, \tau_{t}(Q) \psi\right) \\
=-\int_{0}^{t}\left(\psi, \tau_{s}\left(\tilde{V}^{\prime}(Q)\right) \psi\right) f(s) d s+f(0)(\psi, P \psi)-\left[f^{\prime}(0) M-\int_{0}^{\infty} f(s) \gamma(s) d s\right] \\
\cdot(\psi, Q \psi)+\int_{0}^{t}(\psi, W(s) \psi) f(s) d s
\end{aligned}
$$

where $\tilde{V}$ is the nonlinear part of $V$, i.e. $\tilde{V}(x)=\int_{\mathbb{R}} \exp i \lambda x d v(\lambda)($ see $(2.3))$.

Now, if we make the obvious choice $f(s)=\varphi_{t}(s)$, we get that

$$
\left(\psi, \tau_{t}(Q) \psi\right)=\left(\psi,\left\{B\left(\varphi_{t}\right)-M^{-1} \int_{0}^{t} \varphi_{t}(s) \tau_{s}\left(\tilde{V}^{\prime}(Q)\right)\right\} \psi\right) \quad \forall \psi \in D\left(H^{V}\right) .
$$

But $D\left(H^{V}\right) \supset S\left(\mathbb{R}^{2 N+1}\right)$ is contained in the domains of all the operators involved and is a domain of essential self-adjointness for $\tau_{t}(Q)$. This means that the groups generated by the self-adjoint operators $\tau_{t}(Q)$ and $B\left(\varphi_{t}\right)-M^{-1} \int_{0}^{t} \varphi_{t}(s) \tau_{s}\left(\tilde{V}^{\prime}(Q)\right) d s$ are the same. Thus $\tau_{t}\left(e^{i \mu Q}\right)$ satisfies our equation. Moreover one can check, with the same argument we will employ in Lemma 5.5, that the equation has a unique solution.

Using Eq. (4.5) we obtain the following integral equation for $Q(t)=\tau_{t}(Q)$ :

$$
M Q(t)=\int_{0}^{t} d s \varphi_{t}(s) W(s)+\varphi_{t}(0) P+\left\{\int_{0}^{t} d s \gamma(s) \varphi_{t}(s)-M \varphi_{t}^{\prime}(0)\right\} Q-\int_{0}^{t} d s \varphi_{t}(s) \tilde{V}^{\prime}(Q(s)) .
$$

Quantum Langevin Equation ( $Q L E)$. It can be shown that the classical analog of Eq. (4.7) converges to the integral form of the CLE, if we thermalize the particles in the bath and take the scaling limit. To be more specific, in this limit the objects $F(t)$, the classical analog of $W(t)$, becomes the classical white noise, while $\gamma(t)$ converges to $\eta \delta(t)$ with $\eta=2 \sqrt{k^{*} m^{*}}$ and $\varphi_{t}$ converges to the square integrable function $\varphi_{t}^{\infty}$, defined in $(1.4 \mathrm{~b})$. 
We want to proceed in a similar fashion in the quantum mechanical situation. The immediate difficulty we encounter is, as already noted before, just what is the analog of "we thermalize the particles in the bath?" To repeat again, what are the initial states, $\omega_{0}$, i.e. density matrices on $T$, for which one can prove a "weak" convergence of (4.7) in the scaling limit to what would be an integral form of the QLE (1.3)? To answer this question precisely it is unfortunately necessary to essentially reformulate many notions of classical probability theory in an algebraic way. We do this in Sect. 5 following the works of Winnink [19] and of Accardi, Frigerro and Lewis [18]. Since that work is rather technical we summarize the main conclusion here and also discuss again the reason why choosing $\omega_{0}$ to be a product state doesn't work.

Definition 4.3. To write the integral form of the QLE (1.3) in a precise way we shall consider a Hilbert space $\mathscr{H}$, a set $\{B(f)\}_{f \in \mathscr{R}\left(\mathbb{R}^{+}\right)}$of self-adjoint operators, with common domain of essential self-adjointness $D$, and a state $\tilde{\omega}$ on the algebra of linear operators in $\mathscr{H}$ such that $\exp [i B(f)] \exp [i B(g)]$

$$
\begin{aligned}
& =\exp \left\{i h M^{-1}\left[g^{\prime}(0) f(0)-f^{\prime}(0) g(0)+2 \eta M^{-1}\left(g(0) f(0)+\int_{0}^{\infty} f(s) g^{\prime}(s) d s\right)\right]\right\} \\
& \cdot \exp [i B(g)] \exp [i B(f)], \quad \forall f, g \in \mathscr{B}\left(\mathbb{R}^{+}\right), \\
& \begin{aligned}
\tilde{\omega}(B(f) B(g)+B(g) B(f))= & h \eta M^{-2} \\
& \cdot \int_{\mathbb{R}} x \operatorname{coth}(h \beta x / 2) \hat{f}(x) \hat{g}(x)^{*} d x \forall f, \quad g \in \mathscr{B}_{0}\left(\mathbb{R}^{+}\right),
\end{aligned}
\end{aligned}
$$

where " " is the Fourier transform. Furthermore $\tilde{\omega}$ is a quasi-free (Gaussian) state on the subalgebra generated by $\left\{e^{i B(f)}\right\}_{\in \in \mathscr{B}_{0}\left(\mathbb{R}^{+}\right)}$. The $B(f)^{\prime}$ 's are to be thought of as a smoothed version of the quantum noise operators $W(t)$ defined in $(1.3)$ and $\tilde{\omega}$ as the state with respect to which expectation values are taken there. The position operators $\{Q(t)\}_{t \in \mathbb{R}^{+}}$will undergo a Quantum Ornstein-Ulenbeck (QOU) process if they have a common domain of essential self-adjointness $D$ and they satisfy, with respect to the state $\tilde{\omega}$,

$$
Q(t)=B\left(\varphi_{t}^{\infty}\right)+\int_{0}^{t} \tilde{V}^{\prime}(Q(s)) \varphi_{t}^{\infty}(s) d s,
$$

where $\varphi_{t}^{\infty} \in \mathscr{B}\left(\mathbb{R}^{+}\right)$is a solution of

$$
\left\{\begin{array}{l}
M\left(\varphi_{t}^{\infty}\right)^{\prime \prime}(s)-\eta\left(\varphi_{t}^{\infty}\right)^{\prime}(s)+K \varphi_{t}^{\infty}(s)=0 \quad s \in[0, t] \\
\varphi_{t}^{\infty}(t)=0 ; \quad\left(\varphi_{t}^{\infty}\right)^{\prime}(t)=-1 ; \quad \varphi_{t}^{\infty}(s)=0 \quad s>t .
\end{array}\right.
$$

Remark 4.1. Note that $\tilde{\omega}$ is not completely defined by (4.9) because there exists $f$ such that $f \in \mathscr{B}\left(\mathbb{R}^{+}\right)$but not $f \in \mathscr{B}_{0}\left(\mathbb{R}^{+}\right)$(e.g. $Q(0)=B\left(\varphi_{0}^{\infty}\right)$ ). The choice of $\tilde{\omega}$, compatible with (4.9), corresponds to the choice of the initial conditions that are, in this sense, buried in the state.

The solution of Eq. (4.10) has to be considered with "respect to the state $\tilde{\omega}$ " in the same sense in which the solution of the classical Langevin equation is 
considered with respect to the Wiener process. This also implies that the natural topology in which to understand (4.10) is the topology generated by the state $\tilde{\omega}$ itself through the standard norm $\|A\|_{\tilde{\omega}}^{2}=\tilde{\omega}\left(A^{*} A\right)$. As in the classical case this remark is not particularly relevant in cases, like the one at hand, in which the drift is not random.

Our convergence theorem provides a class of quasi-free states for which the QLE may be solved. There are certainly other states for which the QLE may be solved, but they are more difficult to characterize. We note however that for the often considered product states, where the particle is initially decoupled from the bath, the scaling limit does not exist, so these are not states for which the QLE may be solved. To see this considered the product state $\omega$ with density matrix,

$$
C \rho \otimes \exp -\beta\left\{\sum_{i=1}^{2 N} \frac{p_{i}^{2}}{2 m}+\frac{1}{2} \sum_{i=1}^{2 N} \lambda_{i}^{2} q_{i}^{2}\right\},
$$

where $\rho$ is a density matrix for the zero particle alone and $C$ is a normalization constant. We can rewrite (4.7) as

$$
M Q(t)+\int_{0}^{t} d s \varphi_{t}(s) V^{\prime}(Q(s))=\int_{0}^{t} d s \varphi_{t}(s) \underline{W}(t)+\varphi_{t}(0) P-M \varphi_{t}^{\prime}(0) Q
$$

where

$$
\underline{W}(t)=W(t)+\gamma(t) Q .
$$

$\underline{W}(t)$ now plays the role of the quantum noise as can be readily checked using the results of Appendices I and II. The divergence due to the noise term is now however no longer compensated by a divergence due to the momentum. Assuming that $\tilde{V}^{\prime}(x)$ is bounded, it is then straightforward to check that

$$
\lim _{L \rightarrow \infty} \lim _{N \rightarrow \infty} \omega(Q(t) Q(s))=+\infty \quad \forall t, s>0 .
$$

The product states (4.12) therefore do not lead to a reasonable dynamics in the scaling limit. While (4.14) might seem strange at first, it is easily explained if we go back to our initial Hamiltonian (2.5). The state (4.12) describes a bath of independent harmonic oscillator. In the limit we consider however, the zeroth particle is very strongly coupled with (and strongly couples) the bath variables. Hence the product state corresponds to a state with a large amount of energy, which in the scaling limit gives rise to the singular dynamics.

Our main theorem is then:

Theorem 4.1. The quantum stochastic process determined by the Hamiltonian (2.5) and the state (2.6) converge in the limit $N, L \rightarrow \infty$, to the solution of the QLE with a quasi-free state $\tilde{\omega}$ such that $\forall f, g \in \mathscr{B}\left(\mathbb{R}^{+}\right)$

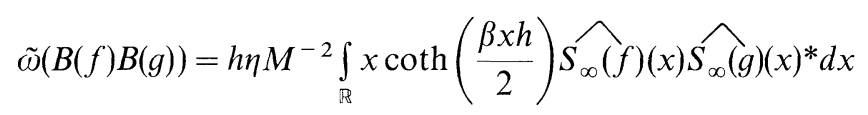

with $S_{\infty}$ as defined in Lemma III.3. The convergence is in the sense of finite dimensional distributions.

We note that the concepts of convergence of Quantum Stochastic Processes 
(QSP), are not standard as yet. In the literature convergence of QSP is normally interpreted as convergence of moments or as convergence of finite dimensional distributions and a reconstruction theorem [18] is sometimes used to produce the limit state. This is clearly a more limited sense of convergence than the classical concept of weak convergence. The importance of considering observables for which the convergence of the finite dimensional distributions is not adequate may be questionable, from a physical point of view. Still more strong or flexible concepts of convergence for QSP may be necessary for technical reasons and we think it is important to try to clarify what can be done in this direction. This is necessary also if we want to compare classical and quantum results. In particular we feel it would be interesting to have for QSP a concept of convergence that reduces to weak convergence on continuous functions in the classical case. Moreover, in our particular problem, we want to have a setting in which the different approximated dynamical equations can be compared directly with each other. For these reasons we will construct explicitly the algebras of observables for our QSP's, which in the classical context are determined by the choice of the path space and we will study the convergence of states on these algebras.

To prove the theorem we note that (4.10) can be regarded as a way of expressing the position operator, when $\widetilde{V}^{\prime} \neq 0$, in terms of the position operator in the harmonic case. It is therefore natural to show that (4.10) has a solution in some suitable Banach space. This runs into the problem that (4.10) involves unbounded operators. To overcome this we exponentiate the equation, which gives us Eq. (4.5). A more serious difficulty is that the obvious Banach space on which to study (4.5) depends strongly on $N$ and $L$. This forces us to study the convergence of Eq. (4.5) on a rather abstract space formed by the operators $B(f), f \in \mathscr{B}\left(\mathbb{R}^{+}\right)$. In our construction, these operators have no natural commutation relations. Particular commutation relations are selected by a special class of states. The program worked out in the next section consists of the following steps:

i) Construct a $C^{*}$-algebra $\mathscr{W}$, generated by the operators $\exp [i \lambda B(f)]$, which has sufficient structure to do integrations.

ii) Find a suitable Banach space of functions from $\mathbb{R} \times \mathbb{R}^{+} \rightarrow \mathscr{W}$. On this space, define the map

$$
F_{N, L}: B \rightarrow B ;\left(F_{N, L} X\right)(\mu, t)=\exp i\left\{B\left(\varphi_{N, L, t}\right)-i M^{-1} \int_{0}^{t} d s \varphi_{N, L, t}(s) \int d v(\lambda) \lambda X(\lambda, s)\right\} .
$$

iii) Show that this map has a fixed point $X_{N, L}(\lambda, t) \equiv \exp i \lambda Q_{N, L}(t)$.

iv) Show that, on the $C^{*}$-algebra $\mathscr{W}$, $\exp i \mu Q_{N, L}(t)$ converges in some sense to $\exp i \mu Q_{\infty}(t)$, which is the fixed point of the map $F_{\infty}-$ the $\operatorname{map} F_{N, L}$ with $\varphi_{N, L, t}$ replaced by $\varphi_{t}^{\infty}$.

v) Prove that $\tilde{\omega}_{K_{0}}^{\infty}(B(f) B(g)) \equiv \lim _{L \rightarrow \infty} \lim _{N \rightarrow \infty} \tilde{\omega}_{K_{0}}^{N, L}(B(f) B(g))$ exists. This is done in Appendix III. Finally, show that $\forall m, t_{1}, \ldots, t_{m}, \mu_{1}, \ldots, \mu_{m} \in \mathbb{R}$

$$
\lim _{L \rightarrow \infty} \lim _{N \rightarrow \infty} \tilde{\omega}_{K_{0}}^{N, L}\left(\prod_{j=1}^{m} \exp i \mu_{j} Q_{N, L}\left(t_{j}\right)\right)=\tilde{\omega}_{K_{0}}^{\infty}\left(\prod_{j=1}^{m} \exp i \mu_{j} Q_{\infty}\left(t_{j}\right)\right) .
$$




\section{Rigorous Derivation of the Quantum Langevin Equation}

Abstract Preliminaries. In order to circumvent the problem of the representation of our operators we adopt an algebraic point of view. To describe the noise induced by the environment on the system, we introduce the set $\mathcal{N}=\{E(f)\}_{f \in \mathscr{B}\left(\mathbb{R}^{+}\right)}$. Eventually, we are going to identify $E(f)$ with $e^{i B(f)}$.

Following Winnink [19] we mimic algebraically the Kolmogorov reconstruction theorem. The first step is to consider the free associative algebra $\mathscr{A}_{f}$ generated by $\mathscr{N}$ and the identity 1 ; moreover we need to introduce a topology; we do that through the norm

$$
\left\|C 1+\sum_{j=1}^{M} \sum_{k=1}^{M_{j}} \alpha_{j k} \prod_{l=1}^{j} E\left(f_{j k k}\right)\right\|_{0}=\sum_{j=1}^{M} \sum_{k=1}^{M_{j}}\left|\alpha_{j k}\right|+|C|
$$

$\forall M, M_{j} \in \mathbb{N} ; C, \alpha_{j k} \in \mathbb{C} ; f_{j k l} \in \mathscr{B}\left(\mathbb{R}^{+}\right)$such that $\forall j, n, m \in \mathbb{N}(n \neq m) \exists l \in \mathbb{N}: f_{j n l} \neq f_{j m l}$.

Clearly (5.1) is a Banach norm, so the closure $\overline{\mathscr{A}}_{f}$ is a Banach algebra. We need now to introduce a minimum of structure. We define the involution generated by

$$
\begin{aligned}
E(f)^{*} & =E(-f) & & \forall f \in \mathscr{B}\left(\mathbb{R}^{+}\right) \\
(C 1)^{*} & =\bar{C} 1 & & \forall C \in \mathbb{C} \\
(A B)^{*} & =B^{*} A^{*} ;(A+B)^{*}=A^{*}+B^{*} & & \forall A, B \in \overline{\mathscr{A}}_{f} .
\end{aligned}
$$

Now we consider, in the Banach *-algebra $\overline{\mathscr{A}}_{f}$, the smallest closed two sided *-ideal $I_{0}$ generated by the elements $(E(0)-1)$ and $E(\lambda f) E(\mu f)-$ $E((\lambda+\mu) f) \lambda, \mu \in \mathbb{R} ; f \in \mathscr{B}\left(\mathbb{R}^{+}\right)$.

Taking the quotient $\mathscr{A}_{f}$ with respect to $I_{0}$ and equipping it with the natural norm we get the new Banach*-algebra $\mathscr{A}_{1}=\overline{\mathscr{A}}_{f} / I_{0}$. The algebra $\mathscr{A}_{1}$ is however far too small for our purposes. In order to overcome this we devise a general strategy to introduce weak norms on $\mathscr{A}_{1}$.

Lemma 5.1. Let $\mathscr{M} \subseteq \mathscr{A}_{1}^{*}$ (the dual of $\mathscr{A}_{1}$ ) be a convex set with the following properties: for each $\omega \in \mathscr{M}$,

i) $\omega(1)=1$

ii) $\omega\left(A^{*} A\right) \geqq 0$ for each $A \in \mathscr{A}_{1}$

iii) $A \in \mathscr{A}_{1}, \quad \omega\left(A^{*} A\right) \neq 0 \Rightarrow \omega_{A} \in \mathscr{M}$, where the state $\omega_{A}$ is defined by $\omega_{A}(B)=\omega\left(A^{*} B A\right) / \omega\left(A^{*} A\right)$ for each $B \in \mathscr{A}_{1}$.

Then

$$
\|A\|_{\mathscr{M}}=\sup _{\omega \in \mathscr{M}} \sqrt{\omega\left(A^{*} A\right)}
$$

is a $C^{*}$-seminorm on $\mathscr{A}_{1}$. Moreover if we set $I_{1}=\left\{A \in \mathscr{A}_{1} \mid\|A\|_{\mathscr{M}}=0\right\}$, and define $\mathscr{A}_{\mathscr{M}}=\mathscr{A}_{1} / I_{1}$, where the closure is taken with respect to the norm $\|\cdot\|_{\mathscr{M}}$ induced on the quotient space by $\|\cdot\|_{\mathscr{k}}$, we have that $\mathscr{A}_{\mathscr{M}}$ is a $C^{*}$-algebra.

Proof. The triangle inequality follows from the Cauchy-Schwarz inequality for positive functionals $\left(\left|\omega\left(A^{*} B\right)\right|^{2} \leqq \omega\left(A^{*} A\right) \omega\left(B^{*} B\right)\right)$. Moreover if $\omega\left(A^{*} A\right)=0$ then 
$\omega\left(A^{*} B^{*} B A\right)=0$ for each $B \in \mathscr{A}_{1}$ so, calling $\mathscr{M}_{A}=\left\{\omega \in \mathscr{M} \mid \omega\left(A^{*} A\right) \neq 0\right\}$ we have

$$
\|B A\|_{\mathscr{\sim}}^{2}=\sup _{\omega \in \mathscr{M}_{A}} \frac{\omega\left(A^{*} B^{*} B A\right)}{\omega\left(A^{*} A\right)} \omega\left(A^{*} A\right) \leqq\|A\|_{\mathscr{M}}^{2}\|B\|_{\mathscr{M}}^{2} .
$$

Next, using Cauchy-Schwarz again,

$$
\|A\|_{\mathscr{M}}^{4} \leqslant \sup _{\omega \in \mathscr{M}} \omega\left(A^{*} A A^{*} A\right) \leqq\left\|A^{*} A\right\|_{\mathscr{M}}^{2} .
$$

Combining (5.2) and (5.3) we find that $\|A\|_{\mathscr{M}} \leqq\left\|A^{*}\right\|_{\mathscr{M}}$. Since this holds for any $A$, this implies that $\|A\|_{\mathscr{M}}=\left\|A^{*}\right\|_{\mathscr{M}}$ and, again using (5.2), (5.3), $\left\|A^{*} A\right\|_{\mathscr{M}}=\|A\|_{\mathscr{M}}^{2}$. Finally it is easy to check that $I_{1}$ is a closed two

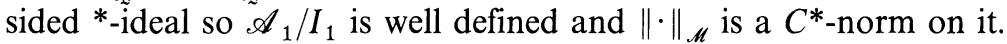

Remark 5.1. The seminorms $\|\cdot\|_{\mathscr{M}}$ are similar to the seminorms used to define the Mackey topology $\tau\left(\mathscr{A}_{1}, \mathscr{A}_{1}^{*}\right)$ (see [20]), the main conceptual difference being that we do not require $\mathscr{M}$ to be closed.

The next thing we need in order to represent the dynamics in our algebra, is a theory of integration adapted to our purposes. We introduce, for each closed $J \subset \mathbb{R}^{n}$, the algebra of functions

$$
\begin{aligned}
C_{\mathscr{M}}^{0}(J)= & \left\{f: J \subset \mathbb{R}^{n} \rightarrow \mathscr{A}_{\mathscr{M}} /\|f\|_{\infty}<\infty ; \forall \omega \in \mathscr{M}, t \in J, \varepsilon \in \mathbb{R}^{+}, \exists \delta \in \mathbb{R}^{+}:\right. \\
& \left.\omega\left([f(t+h)-f(t)]^{*}[f(t+h)-f(t)]\right)<\varepsilon \quad \forall h \in \mathbb{R}^{n},\|h\|<\delta\right\},
\end{aligned}
$$

where

$$
\|f\|_{\infty}=\sup _{t \in J}\|f(t)\|_{\mathscr{M}} .
$$

We define now a weak form of Pettis integral.

Let $\underline{M}=\overline{\operatorname{span}(\mathscr{M})}$, where the closure is taken with respect to the norm topology, then $\forall f \in C_{\mathscr{M}}^{0}(J)$ and any finite measure $v$ on $J$ we define $\int_{J} f d v$ to be the element of $\underline{M}^{*}$ defined by

$$
\omega\left(\int_{J} f d v\right)=\int_{J} \omega(f) d v \quad \forall \omega \in \mathscr{M} .
$$

This definition clearly has the drawback that the integral can fail to belong to $\mathscr{A}_{\mathscr{M}}$. For this reason we are going to enlarge our algebra again (we get in this way the classical analogue of the norm closure of the observable involving only finitely many times).

$$
\mathscr{W}_{0}=\left\{A \in \underline{M}^{*} / \exists J \subset \mathbb{R}^{n} \text { closed, } f \in C_{\mathscr{M}}^{0}(J), v \text { finite measure on } J: A=\int_{J} f d v\right\} .
$$

The preceding definition is justified by the following.

Lemma 5.2. $\mathscr{W}=\overline{\mathscr{W}}_{0}$ is a $C^{*}$-algebra.

Proof. We start noticing that property (iii) in Lemma 5.1 implies that if $\omega \in \mathscr{M}$, then $\omega(A \cdot)$, for each $A \in \mathscr{A}_{\mathscr{M}}$, is an element of $\underline{M}$. This implies that, if $\int_{J_{1}} f_{1} d v_{1}=$ 
$\int_{J_{2}} f_{2} d v_{2}$, then $\int_{J_{1}} A f_{1} d v_{1}=\int_{J_{2}} A f_{2} d v_{2} \forall A \in \mathscr{A}_{\mathcal{M}}$. In fact the latter is certainly true if $v_{1}$ and $v_{2}$ are finite linear combinations of point mass measures, if not then they can be approximated via a sequence of point masses measures $v_{i}^{(n)}, i \in\{1,2\}$, so, for each $\omega \in \mathscr{M}$, we have

$$
\omega\left(\int_{J_{i}} f_{i} d v_{i}\right)=\int_{J_{i}} \omega\left(f_{i}\right) d v_{i}=\lim _{n \rightarrow \infty} \int_{J_{i}} \omega\left(f_{i}\right) d v_{i}^{(n)}
$$

and

$$
\omega\left(\int_{J_{1}} A f_{1} d v_{1}\right)=\lim _{n \rightarrow \infty} \int_{J_{2}} \omega\left(A f_{2}\right) d v_{2}^{(n)}=\omega\left(\int_{J_{2}} A f_{2} d v_{2}\right) .
$$

What we have seen so far is enough to show that if $A_{i}=\int_{J_{l}} f_{i} d v_{i} \in \mathscr{W}_{0} i \in\{1,2\}$ then the product

$$
A_{1} A_{2}=\int_{J_{1} \times J_{2}} f_{1} f_{2} d\left(v_{1} \times v_{2}\right)
$$

is well defined.

To check this let $A_{i}=\int_{L_{i}} f_{i} d \underline{v}_{i}$, then $\forall \omega \in \mathscr{M} \quad \omega\left(\int_{L_{1} \times L_{2}} f_{-} f_{2} d v_{1} \times v_{2}\right)=$ $\int_{\underline{J}_{1}} \omega\left(\int_{\underline{J}_{2}} f_{1} f_{2} d \underline{v}_{2}\right) d \underline{v}_{1}=\int_{J_{1}} \omega\left(\int_{J_{2}} f_{1} f_{2} d v_{2}\right) d \underline{v}_{1}=\int_{J_{2}} \omega\left(\int_{L_{1}} f_{1} f_{2} d \underline{v}_{1}\right) d v_{2}=$ $\omega\left(\int_{J_{1} \times J_{2}} f_{1} f_{2} d v_{1} \times v_{2}\right)$, where we have used Fubini theorem and exchanged the order of integration.

Remark 5.2. Lemma 5.2 could be avoided noticing that $\mathscr{M}^{*}$ is a von Neumann algebra. This can be seen by using a construction similar to the one used to produce the universal enveloping algebra of $A_{\mathscr{M}}$. Unfortunately $\underline{M}^{*}$ is too big for our purposes if $\underline{M} \neq \overline{\mathscr{M}}$, where the closure is in the weak sense, (essentially $\mathscr{M}^{*}$ corresponds, in the classical case, to the algebra of all bounded measurable functions). Note that in the classical (commutative) case it is possible to choose $\mathscr{M}$ weakly-* closed (allowing us to work in $\underline{M}^{*}$ ) using the various characterizations of tightness. At this stage however our understanding of compact sets in the non-commutative case is not sufficient to proceed here in the same manner.

Before the next lemma we define $S \in C_{\mathscr{M}}^{0}(\mathbb{R})$ to be a $\mathscr{M}$-continuous unitary one parameter group of operators if

$$
\begin{aligned}
S(0) & =1, \\
S(-\lambda)^{*} & =S(\lambda), \\
S(\lambda) S(\mu) & =S(\lambda+\mu) \quad \forall \lambda, \mu \in \mathbb{R} .
\end{aligned}
$$

Lemma 5.3. For each $\mathscr{M}$-continuous unitary one-parameter group $S$ we introduce $\operatorname{Exp}_{S}: \mathscr{W} \rightarrow C_{\mathscr{M}}^{0}(\mathbb{R})$, defined as follows: $\forall A \in \mathscr{W}, \operatorname{Exp}_{S}(A)$ is the unique solution of the equation

$$
X(\lambda)=S(\lambda)+i \int_{0}^{\lambda} S(\lambda-\sigma) A X(\sigma) d \sigma \quad \forall \lambda \in \mathbb{R}
$$


If $A^{*}=A, \operatorname{Exp}_{S}(A)$ is a unitary $\mathscr{M}$-continuous one-parameter group, and for each self-adjoint $A, B \in \mathscr{W}$ the following estimate holds:

$$
\left\|\operatorname{Exp}_{S}(A)(\lambda)-\operatorname{Exp}_{S}(B)(\lambda)\right\|_{\mathscr{M}} \leqq|\lambda|\|A-B\|_{/} .
$$

Proof. We will prove the first part of the lemma showing the existence of a unique fixed point for the maps $F_{\Lambda}: C_{\mathscr{M}}^{0}([-\Lambda, \Lambda]) \rightarrow C_{\mathscr{M}}^{0}([-\Lambda, \Lambda]) \Lambda \in \mathbb{R}^{+}$ defined by

$$
F_{\Lambda}(f)(\lambda)=S(\lambda)+i \int_{0}^{\lambda} S(\lambda-\sigma) A f(\sigma) d \sigma \quad \forall \lambda \in[-\Lambda, \Lambda] \quad f \in C_{\mathscr{M}}^{0}([-\Lambda, \Lambda]) .
$$

The first thing to notice is that, by induction

$$
\begin{gathered}
\left\|F_{\Lambda}^{n}(f)\right\|_{\infty} \leqq \sum_{k=0}^{n-1}\left(\frac{\|A\|_{\mathscr{M}}^{k} \Lambda^{k}}{k !}\right)+\frac{\|A\|_{\mathscr{M}}^{n} \Lambda^{n}}{n !}\|f\|_{\infty} \leqq e^{\Lambda\|A\|_{\mathscr{M}}}+\frac{\Lambda^{n}\|A\|_{\mathscr{M}}^{n}}{n !}\|f\|_{\infty}, \\
\left\|F_{\Lambda}^{n}(f)-F_{\Lambda}^{n}(g)\right\|_{\infty} \leqq\|f-g\|_{\infty} \frac{\Lambda^{n}\|A\|_{\mathscr{M}}^{n}}{n !} \forall f, g \in C_{\mathscr{M}}^{0}([-\Lambda, \Lambda]) .
\end{gathered}
$$

Here we have used $\|S\|_{\infty}=1$.

This implies that, for each $f \in C_{\mathcal{M}}^{0}([-\Lambda, \Lambda])$, the sequence $\left\{F_{\Lambda}^{n}(f)\right\}_{n \in \mathbb{N}}$ is a Cauchy sequence, the limit is independent of $f$, and is the unique fixed point of $F_{\Lambda}$. Since $\Lambda$ is arbitrary we get the result $\forall \lambda \in \mathbb{R}$. Now, from (5.10), it is clear that $\operatorname{Exp}_{S}(A)(\lambda)^{-1}$ exist for small $\lambda$. A direct computation then shows that $\forall \mu \in \mathbb{R}$, $X(\mu)=\operatorname{Exp}_{S}(A)(\mu+\lambda) \operatorname{Exp}_{s}(A)(\lambda)^{-1}$ satisfies (5.10). The group property $(5.9 \mathrm{c}) \forall \mu \in \mathbb{R}$ and $\lambda$ small then follows from the unicity of the solution (5.10). This implies the existence of $\operatorname{Exp}_{S}(A)(\lambda)^{-1}$ for each $\lambda \in \mathbb{R}$ and, consequently, the group property for all $\lambda, \mu$. Last we check that $\operatorname{Exp}_{S}(A)(\lambda)$ is unitary. In order to do so we introduce the adjoint equation

$$
X(\lambda)=S(-\lambda)-i \int_{0}^{\lambda} X(\sigma) A S(\sigma-\lambda) d \sigma=F_{\Lambda}\left(X^{*}\right)(\lambda)^{*}
$$

The same arguments used before show that this equation has a unique solution, clearly the solution is $\operatorname{Exp}_{S}(A)(\lambda)^{*}$. Therefore

$$
\begin{aligned}
& \left\|\operatorname{Exp}_{S}(A)(\lambda)-\operatorname{Exp}_{S}(A)(-\lambda)^{*}\right\|_{\mathscr{M}} \\
& =\left\|\int_{0}^{\lambda} \operatorname{Exp}_{S}(A)(-\lambda+\sigma)^{*} A S(\sigma)-\int_{0}^{\lambda} S(\lambda-\sigma) A \operatorname{Exp}_{S}(A)(\sigma)\right\|_{\mathscr{M}} \\
& \leqq\left\|\int_{0}^{\lambda}\left[\operatorname{Exp}_{S}(A)(-\lambda+\sigma)^{*}-\operatorname{Exp}_{S}(A)(\lambda-\sigma)\right] A S(\sigma)\right\|_{\mathscr{M}} \\
& \quad+\| \int_{0}^{\lambda} d \sigma \int_{0}^{\lambda-\sigma} d \tau S(\lambda-\sigma-\tau) A \operatorname{Exp}_{S}(\tau) A S(\sigma) \\
& \quad-\int_{0}^{\lambda} d \sigma \int_{0}^{\sigma} d \tau S(\lambda-\sigma) A \operatorname{Exp}_{S}(-\sigma+\tau)^{*} A S(\tau) \|_{\mathscr{M}} \\
& \quad+\left\|\int_{0}^{\lambda} S(\lambda-\sigma) A\left[\operatorname{Exp}_{S}(A)(\sigma)-\operatorname{Exp}_{S}(A)(-\sigma)^{*}\right]\right\|_{\mathscr{M}} .
\end{aligned}
$$


It is then easy to show that when $\lambda$ is restricted to be in the interval $[-\Lambda, \Lambda]$,

$$
\begin{aligned}
\left\|\operatorname{Exp}_{S}(A)^{*}-\operatorname{Exp}_{S}(A)^{-1}\right\|_{\infty} \leqq & 2\|A\|_{\mathscr{M}} \Lambda\left\|\operatorname{Exp}_{S}(A)^{*}-\operatorname{Exp}_{S}(A)^{-1}\right\|_{\infty} \\
& +\frac{\Lambda^{2}}{2}\|A\|_{\mathscr{M}}^{2}\left\|\operatorname{Exp}_{S}(A)^{*}-\operatorname{Exp}_{S}(A)^{-1}\right\|_{\infty} .
\end{aligned}
$$

So $\operatorname{Exp}_{S}(A)(\lambda)^{*}=\operatorname{Exp}_{S}(A)(\lambda)^{-1}$ for small $\lambda$. The result for all values of $\lambda$ now follows from the group property. Finally, formula (5.11) follows from the easy estimate

$$
\left\|\operatorname{Exp}_{S}(A)(\lambda)-S(\lambda)\right\|_{\mathscr{M}} \leqq|\lambda|\|A\|_{. /}
$$

and the equality $\operatorname{Exp}_{\operatorname{Exp}_{S}(B)}(A-B)=\operatorname{Exp}_{S}(A)$ that can be verified, similarly to what we just did, writing the equation satisfied by $\operatorname{Exp}_{\operatorname{Exp}_{S}(B)}(A-B)$ and noticing, after some algebraic manipulation, that it is just (5.10) again.

Remark 5.3. For each representation $(\pi, \mathscr{H})$ continuous with respect to the topo$\operatorname{logy} \sigma\left(\mathscr{W}, \mathscr{M}_{*}\right)$ on $\mathscr{W}$ and the strong topology on $\mathscr{L}(\mathscr{H})$ it is immediate to see that $\pi(S(\lambda)), \pi\left(\operatorname{Exp}_{S}(A)(\lambda)\right)$ are strongly continuous one parameter groups on $\mathscr{H}$. Hence, by Stone's theorem, there is a self-adjoint operator $B$ on $\mathscr{H}$ such that $\pi(S(\lambda))=e^{i B \lambda}$. The content of Lemma 5.3 is then:

$$
\pi\left(\operatorname{Exp}_{S}(A)(\lambda)\right)=e^{i \lambda(B+\pi(A))} .
$$

Application to the Concrete Model. We can now translate the reduced dynamics, Eq. (4.5), into our new language.

We start by defining

$$
\begin{gathered}
\pi_{N, L}: \mathscr{A}_{1} \rightarrow \mathscr{L}\left(L^{2}\left(\mathbb{R}^{2 N+1}\right)\right), \\
\pi_{N, L}(E(f))=e^{i B_{N, L}(f)} \quad \forall f \in \mathscr{B}(\mathbb{R}) .
\end{gathered}
$$

These, clearly, determine representations of the algebra $\mathscr{A}_{1}$. It is now time to choose our $\mathscr{M}$.

$$
\begin{aligned}
\mathscr{M}= & \left\{\tilde{\omega} \in \mathscr{A}_{1}^{*} / \forall A \in \mathscr{A}_{1} ; f \in \mathscr{B}\left(\mathbb{R}^{+}\right) ; \varepsilon \in \mathbb{R}^{+} \exists \delta \in \mathbb{R}^{+}: \tilde{\omega}\left(A^{*} A\right)>0 ; \tilde{\omega}(1)=1 ;\right. \\
& \left.\tilde{\omega}\left(A^{*}[E(f)-E(g)]^{*}[E(f)-E(g)] A\right)<\varepsilon \forall g \in \mathscr{B}\left(\mathbb{R}^{+}\right):\|f-g\|<\delta\right\} .
\end{aligned}
$$

The first result is

\section{Lemma 5.4.}

$$
\tilde{\omega}_{K_{0}}^{N, L}=\left(\pi_{N, L}\right) *\left(\omega_{K_{0}}^{N, L}\right) \in \mathscr{M},
$$

where $\left(\pi_{N, L}\right)^{*}: \mathscr{L}\left(L^{2}\left(\mathbb{R}^{2 N+1}\right)\right)^{*} \rightarrow \mathscr{A}_{1}^{*}$ is defined by $\left(\pi_{N, L}\right)^{*}(\omega)(A)=\omega\left(\pi_{N, L}(A)\right)$ $\forall \omega \in \mathscr{L}\left(L^{2}\left(\mathbb{R}^{2 N+1}\right)\right)^{*}, A \in \mathscr{A}_{1}$ and $\omega_{K_{0}^{N}}^{N, L}$ is the state specified by (2.6). Moreover $\pi_{N, L}$ extends to a representation $\pi_{N, L}: \mathscr{W} \rightarrow \mathscr{L}\left(L^{2}\left(\mathbb{R}^{2 N+1}\right)\right)$.

Proof. During this proof we will drop the script $N, L$. From Appendix III we have

$$
\begin{aligned}
e^{i(B(f)+B(g))} & =e^{i B(f)} e^{i B(g)} e^{i \sigma(f, g) / 2}, \\
\omega_{K_{0}}\left(e^{i B(f)}\right) & =e^{-C(f, f) / 2} .
\end{aligned}
$$


This implies that $\left(\pi^{*} \omega_{K_{0}}\right)(A E(f) E(g) B)=\left(\pi^{*} \omega_{K_{0}}\right)(A E(g+f) B) e^{-i \sigma(f, g) / 2}$, and we can therefore check by induction that $\pi^{*}\left(\omega_{K_{0}}\right) \in \mathscr{M}$. For the second statement we prove first that if $\omega\left(A^{*} A\right)=0 \forall \omega \in \mathscr{M}$ then $\pi(A)=0$. This follows from $0=\left(\pi^{*} \omega_{K_{0}}\right)\left(A^{*} A\right)=$ $\omega_{K_{0}}\left(\pi(A)^{*} \pi(A)\right)$. So we have a representation $\pi$ of $\mathscr{A}_{\mathscr{M}}$ in $\mathscr{L}\left(L^{2}\left(\mathbb{R}^{2 N+1}\right)\right)$. In order to obtain a unique extension on all $\mathscr{W}$ we remark that $\mathscr{A}_{\mathscr{M}}$ is dense in $\mathscr{W}$ in the topology generated by the seminorms $\|A\|_{\omega}^{2}=\omega\left(A^{*} A\right)$. Indeed for each $A \in \mathscr{W}_{0}$, $A=\int_{J} f d v$, we can find a sequence of point masses measures $v_{n}$ on $J$ converging weakly to $v$ (implying that $v_{n} \times v_{n} \Rightarrow v \times v$ ), from which follows that

$$
\lim _{n \rightarrow \infty} \omega\left(\left[\int_{J} f d v-\int_{J} f d v_{n}\right]^{*} \cdot\left[\int_{J} f d v-\int_{J} f d v_{n}\right]\right)=0 .
$$

As a consequence we may extend $\pi$ on $\mathscr{W}$ provided we show that if a net $\left\{A_{\alpha}\right\} \subset \mathscr{W}$ converges to 0 in the preceding topology then $\left\{\pi\left(A_{\alpha}\right)\right\}$ converges to 0 in the strong topology. To see this we first observe that the uniform principle and the theory of representations implies $\left\|\pi\left(A_{\alpha}\right)\right\| \leqq\left\|A_{\alpha}\right\|_{\mathscr{M}}<C$ for each $\alpha$. Moreover, recalling that $\omega_{K_{0}}(\cdot)=\sum_{n}\left(\psi_{n}, \cdot \psi_{n}\right) e^{-\beta E_{n}}$, where $\left\{\psi_{n}\right\}$ is a orthonormal base for $L^{2}\left(\mathbb{R}^{2 N+1}\right)$ and $E_{n} \rightarrow \infty$, we have that $\left(\pi^{*} \omega_{K_{0}}\right)\left(A_{\alpha}^{*} A_{\alpha}\right) \rightarrow 0$ is equivalent to $\lim _{\alpha} \sum_{n}\left(\psi_{n}, \pi\left(A_{\alpha}\right) \psi_{n}\right) e^{-\beta h E_{n}}=0$.

From this we have $\forall \phi \in L^{2}\left(\mathbb{R}^{2 N+1}\right), \phi=\sum_{n} a_{n} \psi_{n}, \varepsilon \in \mathbb{R}^{+}$,

$$
\begin{aligned}
\left\|\pi\left(A_{\alpha}\right) \phi\right\| & \leqq\left\|\left(A_{\alpha}\right)\left(\sum_{n}^{k} a_{n} \psi_{n}\right)\right\|+C \varepsilon_{k} \leqq \sum_{n}^{k}\left|a_{n}\right| e^{-\beta E_{n} h / 2}\left\|\pi\left(A_{\alpha}\right) \psi_{n}\right\| e^{-\beta E_{n} h / 2}+C \varepsilon_{k} \\
& \leqq\left(\sum_{n}^{k}\left|a_{n}\right|^{2} e^{-\beta h E_{n}}\right)^{1 / 2} \varepsilon_{\alpha}+C \varepsilon_{k}<\varepsilon,
\end{aligned}
$$

where we have chosen $\alpha, k$ big enough.

A consequence of the previous lemma is

Lemma 5.5. Let $\varphi_{t}$ be defined as in (4.6) with any regular cut off on the negative axis and let $S_{t}(\lambda)=E\left(\lambda \varphi_{t}\right)$, then the equation

$$
\begin{aligned}
X(\mu, t) & =\operatorname{Exp}_{s_{t}}\left(-i M^{-1} \int_{0}^{t} \varphi_{t}(s)\left[\int_{\mathbb{R}} \lambda X(\lambda, s) d v(\lambda)\right] d s\right)(\mu) \mu, \lambda \in \mathbb{R}, \quad t \in[0, T], \\
X(\mu, t)^{*} & =X(-\mu, t)
\end{aligned}
$$

has a unique solution and $\pi(X(\mu, t))=e^{i \mu Q(t)}$.

Proof. It follows from Remark 5.3 that if $X(\mu, t)$ is a solution of $(5.15)$ then $\pi(X(\mu, t))$ is a solution of (4.5).

The last statement follows then from Lemma 4.2. We are thus left with the task to prove the existence and unicity of the solution of (5.15).

We study the equation in the space $\mathscr{V}_{v}$ defined as the closure of $\left\{f \in C_{\mathscr{M}}^{0}(\mathbb{R} x[0, T]) / f(\lambda, t)^{*}=f(-\lambda, t)\right\}$ with respect to the norm $\|f\|_{v}=$ $\sup \int\|f(\lambda, s)\|_{\mathscr{M}}|\lambda| d v(\lambda)$, notice that if $f \in \mathscr{V}_{v}$ then $\forall \omega \in \mathscr{M}, t \in[0, T], \omega(f(\cdot, t)) \in$ $s \in[0, T] \mathbb{R}$ $L^{1}(\mathbb{R},|\lambda| v(\lambda))$ so the integration is still well defined for this function. 
Let $F_{T}: \mathscr{V}_{v} \rightarrow \mathscr{V}_{v}$ be given by

$$
F_{T}(f)(\mu, t)=\operatorname{Exp}_{s_{t}}\left(-i M^{-1} \int_{0}^{t} \varphi_{t}(s)\left[\int_{\mathbb{R}} \lambda f(\lambda, s) d v(\lambda)\right] d s\right)(\mu) .
$$

The first thing to notice is that $i \int \lambda f(\lambda, s) d v(\lambda)$ is self-adjoint for each $s \in[0, T]$. Lemma 5.3 then implies that $F_{T}: \mathscr{V}_{v}^{\mathbb{R}} \rightarrow \mathscr{V}_{v}$,

and

$$
\left\|F_{T}(f)(\mu, t)\right\|_{\mathscr{M}}=1
$$

$$
\left\|F_{T}^{n}(f)-F_{T}^{n}(g)\right\|_{\nu} \leqq \frac{M^{-n}}{n !}\left[\int_{0}^{T}\left|\varphi_{T}(s)\right| d s\right]^{n}\left[\int_{\mathbb{R}} \lambda^{2}|d v(\lambda)|\right]^{n}\|f-g\|_{\nu},
$$

from which, after noticing that

$$
\sup _{t \in[0, T]}\left\|F_{T}(f)(\mu, t)-F_{T}(g)(\mu, t)\right\|_{\mathscr{M}} \leqq|\mu| M^{-1}\left(\int_{0}^{T}\left|\varphi_{T}(s)\right| d s\right)\|f-g\|_{v},
$$

the result follows.

We can now study the limits we are interested in. The thermodynamic limit is well understood, the existence of the dynamics for the full system is known [22], so we will not treat it explicitly, although we could handle it in the same way as the scaling limit. From now on we will consider the thermodynamic limit already taken and we study the limit $L \rightarrow \infty$ for the reduced dynamics of the heavy particle. The first step is the weak convergence of the states $\tilde{\omega}_{K_{0}}^{L}$.

Lemma 5.6. $\forall A \in \mathscr{W}$ we have

$$
\lim _{L \rightarrow \infty} \tilde{\omega}_{K_{0}}^{L}(A)=\tilde{\omega}_{K_{0}}^{\infty}(A)
$$

where $\tilde{\omega}_{K_{0}}^{\infty} \in \mathscr{M}$ is defined by

$$
\begin{gathered}
\tilde{\omega}_{K_{0}}^{\infty}(E(f))=e^{-C_{\infty}(f, f)} \\
\tilde{\omega}_{K_{0}}^{\infty}(A E(f) E(g) B)=(A E(f+g) B) e^{-i \sigma_{\infty}(f, g)} \quad \forall A, B \in \mathscr{W}, f, g \in \mathscr{B}\left(\mathbb{R}^{+}\right)
\end{gathered}
$$

with $C_{\infty}, \sigma_{\infty}$ as in Appendix III-Lemma III.3.

Proof. It is easy, using the results of Lemma III, to check the convergence of $\tilde{\omega}_{K_{0}}^{L}(A)$ for each $A \in \mathscr{A}_{f}$. The theorem follows then from the fact that $\overline{\mathscr{A}}_{f}=\mathscr{A}_{\mathscr{M}}$ and the extension to $\mathscr{W}$ is a consequence of the Lebesgue dominated convergence theorem.

We are now ready to prove the results we are interested in.

The Harmonic Case. As we already noticed, if the potential is harmonic $(v \equiv 0)$ Eq. (5.15) tells"us that the one parameter group generated by the position of the particle at time " $t$ " is given by $E\left(\lambda \varphi_{t}^{L}\right)$. Classically, in this situation, we have the weak convergence of the stochastic process determined by the reduced dynamics to the Ornstein-Uhlenbeck process. We can do something very similar (although weaker) in the present context. We introduce the algebra of observables for the zero particle using the same abstract construction used before for the observables of the noise, with the following changes, $\mathscr{N}_{B}=\left\{E_{B}(f)\right\}_{f \in \mathscr{B}_{1}}$, where $\mathscr{B}_{1}$ is the 
closure in the norm topology of finite linear combinations of point mass measures on the interval $[0, T], \mathscr{A}_{1}^{B}$ is constructed as $\mathscr{A}_{1}$. Further we take

$$
\begin{aligned}
\mathscr{M}_{B}= & \left\{\omega \in\left(\mathscr{A}_{1}^{B}\right)^{*} / \forall A \in \mathscr{A}_{1}^{B} ; t \in[0, T], \lambda \in \mathbb{R}, \varepsilon \in \mathbb{R}^{+} \exists \delta \in \mathbb{R}^{+}: \omega\left(A^{*} A\right) \geqq 0 ; \omega(1)=1 ;\right. \\
& \omega\left(A^{*}\left[E_{B}\left(\lambda \delta_{t}\right)-E_{B}\left(\mu \delta_{s}\right)\right]^{*}\left[E_{B}\left(\lambda \delta_{t}\right)-E_{B}\left(\mu \delta_{s}\right)\right] A\right) \leqq \varepsilon \forall \mu \in \mathbb{R}, s \in[0, T]: \\
& |\lambda-\mu|+|t-s|<\delta\} .
\end{aligned}
$$

We have now the right space to study the convergence of our process to the "Quantum Ornstein-Uhlenbeck" process. In order to study our problem in this new space we define $\pi_{B}^{L}: \mathscr{W}^{B} \rightarrow \mathscr{W}$ through $\pi_{B}^{L}\left(E_{B}\left(\delta_{t}\right)\right)=E\left(\varphi_{t}^{L}\right)$ and the requirement that $\pi_{B}^{L}$ be a ${ }^{*}$-homomorphism. It is easy to check that this can be done. So we have

Theorem 5.1. $\hat{\omega}_{K_{0}}^{L}=\left(\pi_{B}^{L}\right)^{*}\left(\tilde{\omega}_{K_{0}}^{L}\right) \stackrel{L \rightarrow \infty}{\longrightarrow} \hat{\omega}_{K_{0}}^{\infty}$ in the $\sigma\left(\mathscr{M}_{B}, \mathscr{W}_{B}\right)$ topology where $\hat{\omega}_{K_{0}}^{\infty} \in \mathscr{M}_{B}$ is defined by $\omega_{K_{0}}^{\infty}\left(E\left(\lambda \delta_{t}\right)\right)=e^{\lambda^{2} C^{\infty}\left(\varphi_{t}^{\infty}, \varphi_{t}^{\infty}\right)}$,

$$
\omega_{K_{0}}^{\infty}\left(A E\left(\lambda \delta_{t}\right) E\left(\mu \delta_{s}\right) B\right)=\hat{\omega}_{K_{0}}^{\infty}\left(A E\left(\lambda \delta_{t}+\mu \delta_{s}\right) B\right) e^{-\lambda \mu \sigma^{\infty}\left(\varphi_{t}^{\infty}, \varphi_{s}^{\infty}\right)} \quad \forall A, B \in \mathscr{W}_{B} .
$$

Proof. Lemma III.2-3 and $\lim _{L \rightarrow \infty}\left\|\varphi_{t}^{L}-\varphi_{t}^{\infty}\right\|=0$ imply immediately that

$$
\lim _{L \rightarrow \infty} \hat{\omega}_{K_{0}}^{L}\left(E\left(\lambda \delta_{t}\right)\right)=\hat{\omega}_{K_{0}}^{\infty}\left(E\left(\lambda \delta_{t}\right)\right) \quad \forall t \in[0, T],
$$

and the same holds for finite products and sums of $E(\lambda \delta)$. We thus have that

$$
\lim _{L \rightarrow \infty} \hat{\omega}_{K_{0}}(A)=\hat{\omega}_{K_{0}}^{\infty}(A), \quad \forall A \in \mathscr{A}_{\mathscr{M}_{B}}
$$

Finally notice that, again by Appendix III, $\left(\pi_{B}^{L}\right)^{*}(\mathscr{M}) \subset \mathscr{M}_{B}$ so if $f \in C_{\mathscr{M}_{B}}^{0}(J)$ we have $\pi_{B}^{L}(f) \in C_{\mathscr{M}}^{0}(J)$ and

$$
\lim _{L \rightarrow \infty} \hat{\omega}_{K_{0}}^{L}\left(\int_{J} f d \mu\right)=\lim _{L \rightarrow \infty} \int_{J} \hat{\omega}_{K_{0}}^{L}(f) d \mu=\int_{J} \hat{\omega}_{K_{0}}^{\infty}(f) d \mu=\hat{\omega}_{K_{0}}^{\infty}\left(\int_{J} f d \mu\right),
$$

where we have used Lebesgue dominated convergence theorem.

Remark 5.4. The content of the theorem is that for a certain class of function $F$ of the observables $Q(t)$ we know $\lim _{L \rightarrow \infty} \lim _{N \rightarrow \infty} \omega_{K_{0}}^{N, L}(F(Q))$. Notice that this class does not contain any polynomials, this situation, however, occurs also in the classical case [11] and it is due to technical not conceptual reasons.

The General Case. We consider the general case as a perturbation of the harmonic one.

First of all we have the analogue of Lemma 5.5. This means that the dynamics is determined by the following equations for $X_{L}(\mu, \cdot) \in C_{{ }^{\prime}}^{0}(\mathbb{R} \times[0, T])$,

$$
\begin{aligned}
X_{L}(\mu, t) & =\operatorname{Exp}_{E_{B}\left(\cdot \delta_{t}\right)}\left(-i M^{-1} \int_{0}^{t} \varphi_{t}^{L}(s)\left[\int_{\mathbb{R}} \lambda X_{L}(\lambda, s) d v(\lambda)\right] d s\right)(\mu) \\
X_{L}(-\mu, t)^{*} & =X_{L}(\mu, t), \quad L \in \mathbb{R}^{+} \cup\{\infty\} .
\end{aligned}
$$

The fundamental fact is given by 


\section{Lemma 5.7.}

$$
\lim _{L \rightarrow \infty}\left\|X_{L}-X_{\infty}\right\|_{\mathscr{M}_{B}}=0
$$

Proof. As we did in Lemma 5.5 we define

$$
F_{T}^{L}(f)(\mu, t)=\operatorname{Exp}_{E_{B}\left(\delta_{t}\right)}\left(-i M^{-1} \int_{0}^{t} \varphi_{t}^{L}(s)\left[\int_{\mathbb{B}} \lambda f(\lambda, s) d v(\lambda)\right] d s\right)(\mu),
$$

so Eq. (5.15) can be rewritten as $X_{L}=F_{T}^{L}\left(X_{L}\right)$. The first thing to notice is that $\forall f \in C_{\mathscr{M}_{B}}^{0}(\mathbb{R x}[0, T])$ with $f(-\mu, t)^{*}=f(\mu, t)$, Lemma 5.3 implies

$$
\begin{aligned}
\left\|F_{T}^{L}(f)(\mu, t)-F_{T}^{\infty}(f)(\mu, t)\right\|_{\mathscr{M}_{B}} & \leqq M^{-1}|\mu| \int_{0}^{t}\left|\varphi_{t}^{L}(s)-\varphi_{t}^{\infty}(s)\right| \int_{\mathbb{R}}|\lambda|\|f(\lambda, s)\|_{\mathscr{M}_{B}}|d v(\lambda)| d s \\
& \leqq M^{-1}\|f\|_{v}\left\|\varphi_{t}^{L}-\varphi_{t}^{\infty}\right\| \int_{\mathbb{R}} \lambda^{2}|d v(\lambda)| .
\end{aligned}
$$

So, using the explicit form of $\varphi_{t}^{L}$, we have

$$
\lim _{L \rightarrow \infty}\left\|F_{T}^{L}(f)-F_{T}^{\infty}(f)\right\|_{v}=0 .
$$

Moreover, in the proof of Lemma 5.5, we have already obtained the estimates

$$
\left\|\left(F_{T}^{L}\right)^{n}(f)-\left(F_{T}^{L}\right)^{n}(g)\right\|_{\nu} \leqq \frac{D^{n}}{n !}\|f-g\|_{\nu},
$$

where we may choose $D$ independent of $L \in \mathbb{R} \cup\{\infty\}$. From all this it follows that $\forall \varepsilon \in \mathbb{R}^{+}$, if $L$ is chosen big enough,

$$
\begin{aligned}
\left\|X_{L}-X_{\infty}\right\|_{\nu} & \leqq\left\|\left(F_{T}^{L}\right)^{n}\left(X_{L}\right)-\left(F_{T}^{L}\right)^{n}\left(X_{\infty}\right)\right\|_{\nu}+\left\|\left(F_{T}^{L}\right)^{n}\left(X_{\infty}\right)-\left(F_{T}^{\infty}\right)^{n}\left(X_{\infty}\right)\right\|_{\nu} \\
& \leqq \frac{D^{n}}{n !}\left\|X_{L}-X_{\infty}\right\|_{v}+\varepsilon
\end{aligned}
$$

so it is enough to choose $D^{n} / n !<1$ to prove that $\lim \left\|X_{L}-X_{\infty}\right\|_{v}=0$ and this implies, using again (5.19), $\lim _{L \rightarrow \infty}\left\|X_{L}-X_{\infty}\right\|_{\mathscr{M}_{B}}=0$.

We can interpret the last lemma in terms of convergence of states, in the spirit of Theorem 5.1. In order to do so we have to construct the quantum stochastic process that describes the position of our particle. For this we denote a copy of the algebra $\mathscr{W}^{B}$ by $\mathscr{W}^{Q}$. In order to describe the observables and to define a quantum stochastic process on it we introduce the maps

$$
\pi_{Q}^{L}: \mathscr{W}^{Q} \rightarrow \mathscr{W}^{B} \text { defined by } \pi_{Q}^{L}\left(E_{Q}\left(\lambda \delta_{t}\right)\right)=X_{L}(\lambda, t) \quad \forall L \in \mathbb{R} \cup\{\infty\} .
$$

This can be done because, again, $\left(\pi_{Q}^{L}\right) *\left(\mathscr{M}_{B}\right) \subset \mathscr{M}_{Q}$.

Theorem 5.2.

$$
\left(\pi_{Q}^{L}\right)^{*}\left(\hat{\omega}_{K_{0}}^{L}\right) \stackrel{L \rightarrow \infty}{\longrightarrow}\left(\pi_{Q}^{\infty}\right)^{*}\left(\hat{\omega}_{K_{0}}^{\infty}\right) \text { in the } \sigma\left(\mathscr{M}_{Q}, \mathscr{W}^{Q}\right) \text { topology. }
$$

Proof. The first step is to show that 


$$
\lim _{L \rightarrow \infty}\left\|\pi_{Q}^{L}(A)-\pi_{Q}^{\infty}(A)\right\|_{\mathscr{M}_{B}}=0 \quad \forall A \in \mathscr{W}^{Q} .
$$

From Lemma 5.7 we have that (5.21) is true for each $A$ that is a finite linear combination of finite products of $E_{Q}\left(\lambda \delta_{t}\right)$. Now, $\pi_{Q}^{L}$ being a representation of $\mathscr{A}_{\mathscr{M}_{Q}}$ in $\mathscr{W}^{B}$, we have $\left\|\pi_{Q}^{L}(A)\right\|_{\mathscr{M}_{B}} \leqq\|A\|_{\mathscr{M}_{Q}} \forall A \in \mathscr{A}_{\mathscr{M}_{Q}}$. This implies that (5.21) is true $\forall A \in \mathscr{A}_{\mathscr{M}_{Q}} \quad$ Finally if $f \in C_{\mathscr{M}_{Q}}^{0}(J)$ then $\pi_{Q}^{L}(f) \in C_{\mathscr{M}_{B}}^{0}(J)$, so $\pi_{Q}^{L}\left(\int_{J} f d \mu\right)=$ $\int_{J} \pi_{Q}^{L}(f) d \mu$ and (5.21) follows from Lebesgue dominate convergence Theorem. Now Theorem 5.1 implies that $\forall A \in \mathscr{W}^{Q}, \varepsilon \in \mathbb{R}^{+}$,

$$
\begin{aligned}
& \left|\left(\pi_{Q}^{L}\right)^{*}\left(\hat{\omega}_{K_{0}}^{L}\right)(A)-\left(\pi_{Q}^{\infty}\right)^{*}\left(\hat{\omega}_{K_{0}}^{\infty}\right)(A)\right| \\
& \quad \leqq\left|\hat{\omega}_{K_{0}}^{L}\left(\pi_{Q}^{L}(A)\right)-\hat{\omega}_{K_{0}}^{L}\left(\pi_{Q}^{\infty}(A)\right)\right|+\left|\hat{\omega}_{K_{0}}^{L}\left(\pi_{Q}^{\infty}(A)\right)-\hat{\omega}_{K_{0}}^{\infty}\left(\pi_{Q}^{\infty}(A)\right)\right| \\
& \quad \leqq\left\|\pi_{Q}^{L}(A)-\pi_{Q}^{\infty}(A)\right\|_{\mathscr{M}_{B}}+\left|\hat{\omega}_{K_{0}}^{L}\left(\pi_{Q}^{\infty}(A)\right)-\hat{\omega}_{K_{0}}^{\infty}\left(\pi_{Q}^{\infty}(A)\right)\right|<\varepsilon
\end{aligned}
$$

if $L$ is big enough.

\section{Solutions of the QLE in the Harmonic Case}

In this section we discuss the behavior of the solutions of the QLE in the case where $V(x)=\frac{1}{2} K x^{2}$. The integrated form of the QLE then becomes very simple, see (5.15):

$$
\exp i \lambda Q(t)=\exp i \lambda B\left(\varphi_{t}\right)
$$

Therefore, the time correlation functions of the position operator of the particle are very easily expressed in terms of the correlation functions of the noise operators $B(f)$. We note that $B(f)$ contains both the usual QN as well as the position and momentum operator of our particle at time zero. For this reason, the expectation value of operators constructed out of the $B(f)$ depend on the initial state. However, as is shown in Appendix III, for a given initial state $\omega_{K_{0}}, B(f)$ can be identified with some integral over the regular $Q N, W(t)$, whose properties are listed in the introduction. More specifically, if we define the operator $S_{\infty}$ as in Appendix III:

$$
S_{\infty}(f)(s)=\left\{f(s)+\theta_{f}^{\infty}(s)\right\}
$$

where $\theta_{f}^{\infty}$ is the square integrable function satisfying

$$
M\left(\theta_{f}^{\infty}\right)^{\prime \prime}(t)-\eta\left(\theta_{f}^{\infty}\right)^{\prime}(t)+K_{0} \theta_{f}^{\infty}(t)=0 ; \quad \theta_{f}^{\infty}(0)=f(0) ; \quad\left(\theta_{f}^{\infty}\right)^{\prime}(0)=f^{\prime}(0),
$$

then, as far as the state $\omega_{K_{0}}$ is concerned, $M B(f)$ can be identified with the regular QN, $W\left(S_{\infty}(f)\right)$, where $W(f) \equiv \int_{-\infty}^{+\infty} d t f(t) W(t), \forall f \in \mathscr{B}(\mathbb{R})$. The $W(f)$ have the following properties:

and

$$
[W(f), W(g)]=-2 \eta h \int_{-\infty}^{+\infty} d k \widehat{f}(-k) \hat{g}(k) k
$$

$$
\left\langle(W(f) W(g)\rangle=2 \eta h \int_{-\infty}^{+\infty} d k \hat{f}(-k) \hat{g}(k) \frac{k}{\exp \beta h k-1} .\right.
$$


We now discuss the following three cases:

i) $K_{0}=K$,

ii) $K_{0} \neq K$ and $K \neq 0$,

iii) $K=0$.

$N$.B. We always have $K_{0}>0$.

Case $i)$ It is easy to check that, in this case,

$$
S_{\infty}\left(\varphi_{t}\right)(s)=\phi(s-t) \equiv\left(T_{t} \phi\right)(s)
$$

(where $\phi$ is as in Lemma 4.2 and $T_{t}$ is the time translation). We then find that

$$
\omega_{K_{0}}(Q(t) Q(s))=\frac{h \eta}{\pi} \int_{-\infty}^{+\infty} d k \frac{\exp i k(s-t) k}{\left[\left(M k^{2}-K\right)^{2}+\eta^{2} k^{2}\right] \exp [\beta h k]-1} .
$$

As expected, $\omega_{K_{0}}$ is a stationary state of the QLE with $V(x)=\frac{1}{2} K x^{2}$. We note that expectation values involving momentum operators can be found by simple differentiations, i.e.

$\omega_{K_{0}}(P(t) P(s))=M^{2} \frac{d}{d t} \frac{d}{d s} \omega_{K_{0}}(Q(t) Q(s))=\frac{h M^{2} \eta}{\pi} \int d k \frac{k^{3} \exp i k(s-t)}{\left[\left(M k^{2}-K\right)^{2}+\eta^{2} k^{2}\right] \exp \beta h k-1}$.

Taking the limit $s \rightarrow t$ in (6.6), we then see that the integrand behaves like $1 / k$ for $k \rightarrow \infty$, which leads to a logarithmic divergence.

Case ii) Since $\phi(s)$ and $\phi^{\prime}(s)$ converges to zero as $S$ tends to minus infinity, one easily checks that $S_{\infty}\left(\varphi_{t}\right) \rightarrow T_{t} \phi$, in the $L^{2}$ sense, as $t \rightarrow+\infty$. Consequently, $\lim \omega_{K_{0}}(Q(t+a) Q(s+a))=\omega_{K}(Q(t) Q(s))$. We thus find that all initial states $\omega_{K_{0}}$ approach, when time tends to infinity, the stationary state $\omega_{K}$.

Case iii) In this case no stationary state exists for the system and the particle diffuses. A straightforward calculation yields that

$$
\begin{aligned}
S_{\infty}\left(\varphi_{t}\right)(k)= & \frac{1}{\sqrt{2 \pi}}\left\{\frac{\exp i k t-1}{i k(i k M+\eta)}-\frac{M}{\eta}\left\{1-\exp \frac{-\eta t}{M}\right\} \frac{K_{0}}{(i k M+\eta)\left(-M k^{2}+i \eta k+K_{0}\right)}\right. \\
& \left.+\frac{\Lambda}{-M k^{2}+i \eta k+K_{0}}\right\} .
\end{aligned}
$$

We are interested in the behavior of $\omega_{K_{0}}(Q(t) Q(s))$ for large $t$ and $s$,

$$
\begin{aligned}
\omega_{K_{0}}(Q(t) Q(s))= & 2 \eta \beta^{-1} \int_{-\infty}^{+\infty} d k \overline{S_{\infty}\left(\varphi_{t}\right)(k)} S_{\infty}\left(\varphi_{s}\right)(k)+2 \eta h \int_{-\infty}^{+\infty} d k \overline{S_{\infty}\left(\varphi_{t}\right)(k)} S_{\infty}\left(\varphi_{s}\right)(k) \\
& \cdot\left\{\frac{k}{\exp \beta h k-1}-1\right\} .
\end{aligned}
$$

The second term on the right-hand side of (6.8) is easily verified to remain finite when $t, s$ tend to infinity, while the main contribution to the first term in (6.8) comes from the first term on the right-hand side of (6.7). Thus for large $s, t$ values 
$\omega_{K_{0}}(Q(t) Q(s))$ is approximately equal to

$$
\frac{2 \eta}{2 \pi \beta} \int_{-\infty}^{+\infty} d k \frac{(1+\exp i k(s-t)-\exp i k s-\exp -i k t)}{k^{2}\left(M k^{2}+\eta^{2}\right)} .
$$

After some elementary analysis, we then find

$$
\lim _{s, t \rightarrow \infty} \frac{\omega_{K_{0}}(Q(t) Q(s))}{\min [s, t]}=\frac{2}{\eta \beta} .
$$

Note that the diffusion constant (6.9) does not depend on $h$. So while the short time behavior depends on the value of $h$, the long time behavior does not.

We remark that (6.9) holds even before taking the scaling limit $L \rightarrow \infty$. This can be verified by the method sketched above, using the results of Appendix III. We also remark that our result (6.9) is a special case of the analysis done by Schramm and Grabert [14]. They study the diffusive behavior for Hamiltonions of the form (2.5) with $V(Q)=0$ and arbitrary coefficients $\lambda_{i}$ and $\delta_{i}$. The type of diffusive behavior depends strongly on the choice of these coefficients.

\section{Appendix I}

In this appendix we carry out some useful canonical transformations and study their properties. The technique employed is modeled after [21]. We first diagonalize the Hamiltonian which determines the initial state.

A. Equilibrium.

$$
H^{V_{0}}=H_{0}+\frac{K_{0}}{2} Q_{0}^{2} ; \quad M>m ; \quad K_{0} \geqq 0 .
$$

We perform the diagonalization in two steps, the first is just a Fourier transform:

$$
Q=\Lambda \sim \xi m^{-1 / 2} ; \quad P=\left(\Lambda^{T}\right)^{-1} \eta m^{1 / 2},
$$

where

$$
\Delta_{k n}=(2 N+1)^{-1 / 2} \exp \left(2 \pi i \frac{k n}{2 N+1}\right) \quad k, n \in\{-N, \ldots, N\},
$$

$Q, P \in \mathbb{R}^{2 N+1}$ and $\xi, \eta \in \mathscr{V}_{N}=\left\{z \in \mathbb{C}^{2 N+1} / z_{0} \in \mathbb{R} . \operatorname{Im}\left(z_{k}+z_{-k}\right)=0, \operatorname{Re}\left(z_{k}-z_{-k}\right)=0\right\}$.

In the new coordinates we have

$$
\begin{aligned}
H^{V_{0}} & =\frac{1}{2}(\eta, \eta)+\frac{\alpha}{2}\left(\eta, \mathscr{P}_{\eta}\right)+\frac{1}{2}\left(\xi, \hat{\Omega}^{2} \xi\right)+\frac{\beta}{2}(\xi, \mathscr{P} \xi), \\
\alpha & =\frac{m}{M}-1 ; \quad \beta=\frac{K_{0}}{m} ; \quad \mathscr{P}_{k n}=\Lambda_{\sim 00}^{2} \\
\hat{\Omega}_{l n} & =\delta_{l n}\left[\frac{2 k}{m}\left(1-\cos \left(2 \pi i \frac{k}{2 N+1}\right)\right)\right]^{1 / 2} \operatorname{sign}(l)=\delta_{l n} \hat{\omega}_{l}
\end{aligned}
$$

Next we look for a new transform 


$$
\begin{array}{ll}
\xi=\hat{\Xi} Q, & \xi, \eta \in \mathscr{V}_{N}, \\
\eta=\hat{\Xi}^{T-1} \underset{\sim}{\pi}, & \underset{\sim}{\mathbb{T}}, \underset{\sim}{\pi \in \mathbb{R}^{2 N+1}},
\end{array}
$$

such that

$$
\begin{aligned}
H^{V_{0}} & =\frac{1}{2}\left\{(\pi, \pi)+\left(\underline{\underline{Q}}, \underline{\mu}^{2} Q\right)\right\} \\
\underline{\mu}_{i j} & =\delta_{i j} \mu_{i}
\end{aligned}
$$

This implies

$$
\hat{\Xi} \hat{\Xi}^{*}=1+\alpha \mathscr{P}, \quad\left(\hat{\Xi}^{*}\right)^{-1} \underline{\mu}^{2} \hat{\Xi}^{-1}=\hat{\Omega}^{2}+\beta \mathscr{P} .
$$

As a consequence we get that

$$
\underline{\mu}^{2}=\hat{\Xi}^{-1}\left(\hat{\Omega}^{2}+\alpha \mathscr{P} \hat{\Omega}^{2}+\rho\right) \hat{\Xi}=\hat{\Xi}^{-1} \hat{T} \hat{\Xi}, \quad \rho=K_{0} / M .
$$

So the first step in order to find a matrix satisfying (I.6) is to diagonalize $\widehat{T}$. Now, if we set $v_{l}^{(j)}=i c_{j}\left(\delta_{j l}-\delta_{-j l}\right) j \in\{-N, \ldots,-1\}$, it is easy to check that

$$
\widehat{T} v^{(j)}=\omega_{j}^{2} v^{(j)}
$$

This gives us $N$ eigenvalues, in order to find the other $N+1$, we will assume $\alpha \hat{\omega}_{k}^{2}+\rho \neq 0$ for each $k \in\{0,1, \ldots, N\}$ (it is however easy to remove such a hypothesis). The components of an eigenvector with eigenvalue $\mu_{j}^{2} \neq \hat{\omega}_{k}^{2} \forall k$ must satisfy the following equation:

$$
v_{k}^{(j)}=\left(-\hat{\omega}_{k}^{2}+\mu_{j}^{2}\right)^{-1}\left(e,\left[\alpha \widehat{\Omega}^{2}+\rho 1\right] v^{(j)}\right) e_{k}, \quad e_{k}=\Lambda_{00} ; \quad j \geqq 0 .
$$

So, if we introduce $\hat{u}(\zeta)_{k}=e_{k}\left(\zeta^{2}-\hat{\omega}_{k}^{2}\right)^{-1}$, we have that $v^{(j)}=c_{j} \hat{u}\left(\mu_{j}\right)$, this and (I.8) implies that $F(\zeta)=1-\left(e,\left[\alpha \widehat{\Omega}^{2}+\rho 1\right] \hat{u}(\zeta)\right)$ has the properties $F\left(\mu_{j}\right)=0$ for each $j$.

The function $F$ is a rational function and it is easy to prove that it has $N+1$ real, positive, distinct zeroes $>0$ and $<\hat{\omega}_{N}^{2}$ this accounts for all the eigenvalue of $\hat{T}$.

It is enough to choose $c_{j} \in \mathbb{R}$ for each $j$ in order to obtain that $\hat{\Xi}\left(\mathbb{R}^{2 N+1}\right)=$ $\mathscr{V}_{N}$. The last thing we need to show is that it is possible to choose $c_{j}$ such that (I.6) are satisfies when $\hat{\Xi}_{k n}=v_{k}^{(n)}$.

We notice that (I.6) implies, after some computation,

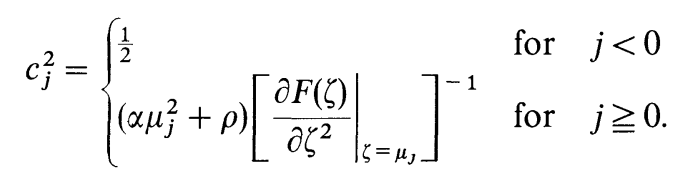

This allows us to obtain the following

Lemma I.1. Let $\hat{\Gamma}$ be the following closed curve on the complex plane:

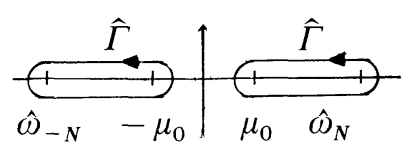

and $g$ any function analytic in the interior of $\hat{\Gamma}$, continuous on $\hat{\Gamma}$ and such that $g(x)=g(-x)$ for each $x \in \mathbb{R}$ then 


$$
\left[\hat{\Xi} g(M) \hat{\Xi}^{*}\right]_{l n}=\frac{1}{2 \pi} \int_{\hat{\Gamma}} \frac{\xi\left(\alpha \zeta^{2}+\rho\right) \hat{u}(\zeta)_{l} \hat{u}(\zeta)_{n} g(\zeta)}{F(\zeta)} d \zeta+\frac{\delta_{l n}}{2 \pi i} \int_{\hat{\Gamma}} \frac{\zeta g(\zeta)}{\zeta^{2}-\hat{\omega}_{k}^{2}} d \zeta
$$

Proof. By direct computation.

Using this formula it is immediate to check that (I.6) hold. Moreover it is useful to notice that, as a consequence of Lemma 1, we have

$$
\left(e, \hat{\Xi} g(M) \hat{\Xi}^{*} e\right)=\frac{m}{2 \pi i M} \int_{\hat{\Gamma}} \frac{\zeta g(\zeta)(e, \hat{u}(\zeta))}{F(\zeta)} d \zeta
$$

$B$. Dynamics. Next we want to find a more convenient form for the Hamiltonian that determines the dynamics:

$$
H^{V}=H_{0}+V\left(Q_{0}\right)
$$

We proceed in analogy with the preceding case. The first canonical transformation is just a useful renumeration:

$$
\begin{array}{llll}
Q=Q_{0} ; & P=P_{0} & & \\
\tilde{q}_{j}=Q_{j} ; & \tilde{p}_{j}=P_{j} \quad-N \leqq j<0 & \tilde{q}=R Q & \\
\tilde{q}_{j}=Q_{j+1} ; & \tilde{p}_{j}=P_{j+1} \quad N>j \geqq 0 & \tilde{p}=R P . & R: \mathbb{R}^{2 N+1} \rightarrow \mathbb{R}^{2 N}
\end{array}
$$

After this we can rewrite

where

$$
H^{V}=\frac{1}{2 m}(\tilde{p}, \tilde{p})+\frac{1}{2 M} P^{2}+\frac{K}{2}(\tilde{q}-e Q, A[\tilde{q}-e Q])+V(Q)
$$

$$
\begin{aligned}
e_{k} & =1 \quad k \in\{-N, \ldots, N-1\}, \\
A_{k n} & =\delta_{k+1, n}-2 \delta_{k, n}+\delta_{k-1, n}+\delta_{k,-1} \delta_{n, 0}+\delta_{k, 0} \delta_{n,-1} .
\end{aligned}
$$

Now we perform, again, a discrete Fourier transformation

$$
\begin{aligned}
Q=Q ; & P=P, \\
\tilde{q}=\Lambda \zeta ; & p=\left(\Lambda^{T}\right)^{-1} \eta, \\
\Lambda_{k n}=(2 N)^{-1 / 2} \exp \left(\pi i \frac{k n}{N}\right) & k, n \in\{-N, \ldots, N-1\},
\end{aligned}
$$

with $\tilde{q}, \tilde{p} \in \mathbb{R}^{2 N}$ and $\xi, \eta \in \tilde{\mathscr{V}}_{N}=\left\{z \in \mathbb{C}^{2 N} / z_{0}, z_{-N} \in \mathbb{R}, \operatorname{Im}\left(z_{k}+z_{k}\right)=0, \operatorname{Re}\left(z_{k}-z_{-k}\right)=0\right\}$.

The Hamiltonian in the new coordinates reads

$$
\begin{aligned}
H^{V} & =\frac{1}{2}\left\{\frac{1}{m}(\eta, \eta)+\frac{1}{M} P^{2}+\left(\xi-Q \Lambda^{*} e, T\left[\xi-Q \Lambda^{*} e\right]\right)\right\}+V(Q), \\
T_{n m} & =\Omega_{n m}^{2}+K \Lambda_{00} \Lambda_{1 n}+K \Lambda_{00} \bar{\Lambda}_{1 m} \\
\Omega_{n m} & =\delta_{n m}\left[2 k\left(1-\cos \left(\pi i \frac{n}{N}\right)\right)\right]^{1 / 2} \operatorname{sign}(n)=\delta_{n m} \omega_{n} .
\end{aligned}
$$

Next we diagonalize $T$, namely we find $\Xi: \mathbb{R}^{2 N} \rightarrow \tilde{\mathscr{V}}_{N}$ such that

$$
\Xi \Xi^{*}=1, \quad \Xi^{-1} T \Xi=\underline{\lambda}^{2} ; \quad \underline{\lambda}_{n m}=\delta_{n m} \lambda_{n} .
$$


Last we use the canonical transformation

$$
\begin{aligned}
Q & =Q ; \quad P=Q, \\
\xi & =\Xi q, \quad \xi, \eta \in \tilde{\mathscr{V}}_{N}, \\
\eta & =\left(\Xi^{T}\right)^{-1} p, \quad q, p \in \mathbb{R}^{2 N},
\end{aligned}
$$

to rewrite the Hamiltonian in the form

$$
\begin{gathered}
H^{V}=\frac{1}{2}\left\{m^{-1}(p, p)+M^{-1} P+\left(q-Q \delta, \underline{\lambda}^{2}[q-Q \delta]\right)\right\}+V(Q), \\
\delta=\Xi^{*} \Lambda^{*} e=\Xi^{*} e_{0} ; \quad\left(e_{0}\right)_{n}=\delta_{n o} \Lambda_{00}^{-1}
\end{gathered}
$$

The equation for the eigenvalue of $T$ reads

$$
\begin{aligned}
\left(\lambda^{2}-\omega_{n}^{2}\right) v_{n} & =k\left[\tau_{n}^{0}\left(\tau^{1}, v\right)+\tau_{n}^{1}\left(\tau^{0}, v\right)\right], \\
\tau_{n}^{0} & =\Lambda_{00} ; \quad \tau_{n}^{1}=\Lambda_{l n}
\end{aligned}
$$

A direct computation shows that (I.18) has no solution for $\lambda^{2}=\omega_{n}^{2}$ $n \in\{-N, \ldots, N-1\}$, so we restrict ourselves to the case $\lambda \neq \omega_{n}$.

If we introduce the vectors $\alpha(\zeta)_{n}=k \tau_{n}^{0}\left(\zeta^{2}-\omega_{n}^{2}\right)^{-1} ; \beta(\zeta)_{n}=k \tau_{n}^{1}\left(\zeta^{2}-\omega_{n}^{2}\right)^{-1}$, we get $v^{(j)}=D_{j} \alpha\left(\lambda_{j}\right)+C_{j} \beta\left(\lambda_{j}\right)$. Substituting this in (I.18) we get a solution if $\underset{\sim}{F}(\zeta)=0$, where

$$
\underset{\sim}{F}(\zeta)=\left|\begin{array}{ll}
\left(\tau^{0}, \beta(\zeta)\right)-1 & \left(\tau^{0}, \alpha(\zeta)\right) \\
\left(\tau^{1}, \beta(\zeta)\right) & \left(\tau^{1}, \alpha(\zeta)\right)-1
\end{array}\right|
$$

Indeed a direct analysis shows that $\underset{\sim}{F}$ has exactly $2 N$ real, positive, distinct zeroes and, more precisely, $\omega_{j}^{2}<\lambda_{2 j}^{2}, \lambda_{2 j+1}^{2}<\omega_{j+1}^{2} j \in\{1, \ldots, 2 N\}$; so we have found all the eigenvalues. The eigenvectors are of the form

$$
\begin{aligned}
v^{(j)} & =c_{j} \chi\left(\lambda_{j}\right), \\
\chi(\zeta) & =\beta(\zeta)+\frac{1-\left(\tau^{0}, \beta(\zeta)\right)}{\left(\tau^{0}, \alpha(\zeta)\right)} \alpha(\zeta) .
\end{aligned}
$$

Notice that the expression (I.20) it is meaningful because the equalities $\left(\tau^{1}, \beta(\zeta)\right)=\left(\tau^{0}, \alpha(\zeta)\right) ;\left(\tau^{1}, \alpha(\zeta)\right)=\left(\tau^{0}, \beta(\zeta)\right)$ imply $\underset{\sim}{F}(\zeta)=\left[1-\left(\tau^{0}, \beta(\zeta)\right)\right]^{2}-\left(\tau^{0}, \alpha(\zeta)\right)^{2}$ and $\left(\tau^{0}, \beta(\zeta)\right)=\left(1-\zeta^{2} / 2 k\right)\left(\tau^{0}, \alpha(\zeta)\right)+\frac{1}{2}$ shows that $\left(\tau^{0}, \alpha(\zeta)\right)=0 \Rightarrow \underset{\sim}{F}(\zeta)=\frac{1}{4} \neq 0$. Finally, being $T$ self-adjoint, if we choose $\Xi_{k n}=v_{k}^{(n)}$ the (I.15) are satisfied, provided that $c_{j}^{2}=1 /\left(\chi\left(\lambda_{j}\right), \chi\left(\lambda_{j}\right)\right)$.

Moreover, it is possible to get a representation of the type given in Lemma I.1 however we need only a specialized form of it.

Lemma I.2. Let $g, \hat{\Gamma}$ be as in Lemma I.1 and $G(\zeta)=\left[1-\left(\tau^{0}, \beta(\zeta)\right)-\left(\tau^{0}, \alpha(\zeta)\right)\right]$, then

$$
\sum_{j=1}^{2 N} \delta_{j}^{2} g\left(\lambda_{j}\right)=\left(e_{0}, \Xi g(\underline{\lambda}) \Xi^{*} e_{0}\right)=\frac{k}{\pi i} \int_{\hat{\Gamma}} \frac{g(\zeta)}{\zeta^{3} G(\zeta)} d \zeta .
$$

Proof. We start noticing that a direct computation yields 


$$
\begin{aligned}
\underset{\sim}{F}(\zeta) & =G(\zeta)\left[1-\left(\tau^{0}, \beta(\zeta)\right)+\left(\tau^{0}, \alpha(\zeta)\right)\right], \\
\delta_{j} & =\frac{c_{j} k}{\lambda_{j}^{2}} \frac{1-\left(\tau^{0}, \beta\left(\lambda_{j}\right)\right)+\left(\tau^{0}, \alpha\left(\lambda_{j}\right)\right)}{\left(\tau^{0}, \alpha\left(\lambda_{j}\right)\right)} .
\end{aligned}
$$

This clearly means that $G(\zeta)=0 \Rightarrow \underset{\sim}{F}(\zeta)=0$ and $\underset{\sim}{F}\left(\lambda_{j}\right)=0, G\left(\lambda_{j}\right) \neq 0 \Rightarrow \delta_{j}=0$ (these are modes that do not interact with the zero particle).

Now, in order to get the formula, we need only to notice that $\partial G(\zeta) /\left.\partial \zeta^{2}\right|_{\zeta=i_{j}^{+}}=$ $\left(\chi\left(\lambda_{j}^{+}\right), \chi\left(\lambda_{j}^{+}\right)\right) 1 / 2 k$, where $\lambda_{j}^{+}$are the zeroes of $G$, and to compute the integral explicitly.

\section{Appendix II}

In this appendix we compute the quantities of interest in the thermodynamic limit taking the limit of the corresponding quantities for the finite system.

\section{Lemma II.1.}

$$
\lim _{N \rightarrow \infty}(\hat{u}(\zeta), e)=\frac{1}{\sqrt{\zeta^{4}-4 \frac{k}{m} \zeta^{2}}}=\frac{1}{D(\zeta)},
$$

where the square root has a cut on $(-2 \sqrt{k / m}, 2 \sqrt{k / m})$ and agree with the arithmetic square root for $\zeta \in \mathbb{R}|\zeta|>2 \sqrt{\mathrm{k} / \mathrm{m}}$ moreover the limit is uniform in $\zeta$ outside each open set containing the cut.

Proof. In fact, clearly we have

$$
\lim _{N \rightarrow \infty}(\hat{u}(\zeta), e)=\frac{1}{2 \pi} \int_{-\pi}^{\pi} \frac{1}{\zeta^{2}-2 \frac{k}{m}(1-\cos \theta)} d \theta=\frac{m}{2 \pi i k} \int_{C} \frac{1}{z^{2}-\left(2 \frac{m}{k} \zeta^{2}\right) z+1} d z
$$

where $c=\{z \in \mathbb{C} /|z|=1\}$. The result follows computing the last integral.

An analogous computation gives us

$$
\begin{aligned}
& \lim _{N \rightarrow \infty} F(\zeta)=\frac{m}{M}-\frac{\alpha \zeta^{2}+\rho}{D(\zeta)}=F_{\infty}(\zeta), \\
& \lim _{N \rightarrow \infty} G(\zeta)=\frac{1}{2}\left[1+D(\zeta / \sqrt{m})(\zeta / \sqrt{m})^{-2}\right] .
\end{aligned}
$$

Finally, we have the equivalent of Lemmas I.1-I.2.

Lemma II.2. In the thermodynamic limit the following hold:

$$
\begin{aligned}
& (e, \hat{\Xi} g(\underline{\mu}) \hat{\Xi} * e)=\frac{m^{2}}{\pi M^{2}} \int_{-2 \sqrt{k / m}}^{2 \sqrt{k / m}} \frac{x^{2} g(x) \sqrt{4 k / m-x^{2}}}{\frac{m^{2}}{M^{2}} x^{2}\left(4 k / m-x^{2}\right)+\left(\alpha x^{2}+\rho\right)^{2}} d x, \\
& \left(e_{0}, \Xi f(\underline{\lambda}) \Xi^{*} e_{0}\right)=\frac{1}{\pi} \int_{-2 \sqrt{k / m}}^{2 \sqrt{k / m}} x^{-2} f(x \sqrt{m}) \sqrt{4 k / m-x^{2}} d x
\end{aligned}
$$


where $x^{2} g(x)$ and $x^{-2} f(x)$ are analytic in a neighborhood of $[-2 \sqrt{k / m}, 2 \sqrt{k / m}]$. Proof. First modify the contour $\hat{\Gamma}$, so that it contains 0 in its interior, and eventually subtract the contribution of the pole at zero. Consequently we can take the limit $N \rightarrow \infty$ inside the integrals using formulas (II.1).

The last step is to shrink the contour on the real axis, remembering the definition of $D(\zeta)$, and taking into account eventual divergences at zero that cancel the therm coming from the modification of $\hat{\Gamma}$.

\section{Appendix III}

Here we compute the quantities of interest for the quantum noise.

Let us recall a few definitions:

$$
\begin{gathered}
W(t)=\sum_{i=1}^{2 N} \lambda_{i}^{2} \delta_{i}\left[\left(q_{i}-Q \delta_{i}\right) \cos \left(\lambda_{i} m^{-1 / 2} t\right)+m^{-1 / 2} p_{i} \lambda_{i}^{-1} \sin \left(\lambda_{i} m^{-1 / 2} t\right)\right] \\
B(f)=f(0) M^{-1} P-\left\{f^{\prime}(0)-M^{-1} \int_{\mathbb{R}^{+}} f(t) \gamma(t) d t\right\} Q+M^{-1} \int_{\mathbb{R}^{+}} W(t) f(t) d t, \quad f \in \mathscr{B}\left(\mathbb{R}^{+}\right) .
\end{gathered}
$$

The first result is

Lemma III.1. Let $\omega_{K_{0}}$ be the state specified by (2.6) with $K_{0}>0$, then we have for each $\psi \in S\left(\mathbb{R}^{2 N+1}\right) ; f, g \in \mathscr{B}\left(\mathbb{R}^{+}\right)$

$$
[B(f), B(g)] \psi=i h \sigma(f, g) \psi,
$$

where

$$
\begin{aligned}
\sigma(f, g)= & M^{-1}\left\{g^{\prime}(0) f(0)-f^{\prime}(0) g(0)\right\} \\
& +M^{-2}\left\{g(0) \int_{\mathbb{R}^{+}} f(t) \gamma(t) d t+\int_{\left(\mathbb{R}^{+}\right)^{2}} f(t) g^{\prime}(s) \gamma(t-s) d t d s\right\}
\end{aligned}
$$

and $h$ is the Planck's constant.

Setting for each $f, g \in \mathscr{B}\left(\mathbb{R}^{+}\right)$

$$
\operatorname{Re}\left\{\omega_{K_{0}}(B(f) B(g))\right\}=C(f, g) .
$$

We have that

$$
|C(f, g)| \leqq D\|f\|\|g\|
$$

with $D \in \mathbb{R}^{+}$independent of $N$. Moreover for each $f, g \in C_{0}^{\infty}\left(\mathbb{R}^{+}\right)$,

$$
\begin{gathered}
C(f, g)=\frac{1}{4 \pi i M} \int_{\hat{\Gamma}} d \zeta \frac{(e, \hat{u}(\zeta)) h \operatorname{coth}(h \beta \zeta / 2)}{F(\zeta)} . \\
\int_{\left(\mathbb{R}^{+}\right)^{2}} e^{-i \zeta(t-s)}\left[f^{\prime \prime}(t)-M^{-1} \int_{t}^{\infty} \gamma(t-\tau) f^{\prime}(\tau)+\rho f(t)\right] \\
{\left[g^{\prime \prime}(s)-M^{-1} \int_{s}^{\infty} \gamma(s-\tau) g^{\prime}(\tau)+\rho g(s)\right] d t d s .}
\end{gathered}
$$


Proof. Formula (III.2) is the result of a straightforward computation. In order to deal with the form $C$ in (III.4) we diagonalize the Hamiltonian defining $\omega_{K_{0}}$, according to Appendix I, through the canonical transformation $\hat{q}=m^{-1 / 2} \Lambda \hat{\Xi} Q$ $\hat{p}=m^{1 / 2} \stackrel{\sim}{\Lambda}^{T-1} \Xi^{T-1} \pi$. We have

$$
\begin{aligned}
& \omega_{K_{0}}\left(Q_{n}\right)=\omega_{K_{0}}\left(\pi_{n}\right)=0, \\
& \omega_{K_{0}}\left({\underset{\sim}{2}}_{n} Q_{m}\right)=\delta_{n m}\left(2 \mu_{n}\right)^{-1} h \operatorname{coth}\left(h \beta \mu_{n} / 2\right), \\
& \omega_{K_{0}}\left(Q_{n} \pi_{m}\right)=\frac{i}{2} \delta_{n m}, \\
& \omega_{K_{0}}\left(\pi_{n} \pi_{m}\right)=\frac{1}{2} \delta_{n m} \mu_{n} h \operatorname{coth}\left(h \beta \mu_{n} / 2\right) .
\end{aligned}
$$

Moreover, according to Appendix I again, we have

$$
\hat{q}_{0}=Q ; \quad \hat{p}_{0}=P ; \quad q=\Xi^{-1} \Lambda^{-1} R \hat{q} ; \quad p=\Xi^{T} \Lambda^{T} R \hat{p} .
$$

This allows us to compute, for each $f, g \in \mathscr{B}\left(\mathbb{R}^{+}\right)$,

$$
\begin{aligned}
& C(f, g)=\left(f(0) m^{1 / 2} M^{-1} \hat{\Xi}^{-1} e\right. \\
& +M^{-1} \hat{\Xi}^{-1} \Lambda^{-1} R^{*} \Lambda \Xi \int_{\mathbb{R}^{+}} \underline{\lambda} \sin \left(m^{-1 / 2} \underline{\lambda} t\right) f(t) d t \delta, \frac{1}{2} \underline{\mu} h \operatorname{coth}(h \beta \underline{\mu} / 2) \\
& \left.\cdot\left[m^{1 / 2} M^{-1} g(0) \hat{\Xi}^{-1} e+M^{-1} \hat{\Xi} \hat{\Xi}^{-1} \Lambda^{-1} R^{*} \Lambda \int_{\mathbb{R}^{+}} \underline{\lambda} \sin \left(m^{-1 / 2} \underline{\lambda} t\right) g(t) d t \delta\right]\right) \\
& +\left(-m^{-1 / 2} f^{\prime}(0) \hat{\Xi}^{*} e+m^{-1 / 2} M^{-1} \hat{\Xi} * \mathcal{\Lambda}^{-1} R^{*} \Lambda \Xi\right. \\
& \cdot \int_{\mathbb{R}^{+}} \underline{\lambda}^{2} \cos \left(m^{-1 / 2} \underline{\lambda t}\right) f(t) \delta, \frac{h}{2 \underline{\mu}} \operatorname{coth}(h \beta \underline{\mu} / 2) \\
& \left.\cdot\left[-m^{-1 / 2} g^{\prime}(0) \hat{\Xi^{*}} e+m^{-1 / 2} M^{-1} \hat{\Xi} * \underset{\sim}{\Lambda}{ }^{-1} R^{*} \Lambda \Xi \int_{\mathbb{R}^{+}} \underline{\lambda}^{2} \cos \left(m^{-1 / 2} \underline{\lambda} t\right) g(t) d t \delta\right]\right) \text {. }
\end{aligned}
$$

This unpleasant formula can be simplified using our knowledge of the dynamics. The idea is to consider the classical evolution determined by the Hamiltonian defining $\omega_{K_{0}}$; on the one hand, performing the transformation defined in Appendix $\mathrm{I}-(A)$, we get

$$
Q(t)=m^{-1 / 2}\left(e_{0}, \Lambda \hat{\Xi}\left\{\cos \underline{\mu} t Q(0)+\underline{\mu}^{-1} \sin \underline{\mu} t \pi(0)\right\}\right) ;
$$

on the other hand the transform defined in Appendix $I-(B)$ leads us to the equation

$$
M Q(t)=-\int_{0}^{t} \gamma(t-s) \dot{Q}(s) d s-K_{0} Q(t)+W(t),
$$

substituting the first expression in the second, and taking into consideration that the equality holds for each initial condition, we obtain the following algebraic relations:

$$
\begin{aligned}
& \left\{-M \underline{\mu}^{2} \cos \underline{\mu} t+\gamma(t) 1-\int_{0}^{t} \gamma(t-s) \underline{\mu} \sin \underline{\mu} s d s+K_{0} \cos \underline{\mu} t\right\} \hat{\Xi}^{*} \Lambda^{*} e_{0} \\
& =\hat{\Xi}^{*} \underline{\Lambda}^{-1} R^{*} \Lambda \Xi \underline{\lambda}^{2} \cos \left(m^{-1 / 2} \underline{\lambda} t\right) \delta,
\end{aligned}
$$




$$
\begin{gathered}
\left\{-M \underline{\mu} \sin \underline{\mu} t+\int_{0}^{t} \gamma(t-s) \cos \underline{\mu} s d s+K_{0} \underline{\mu}^{-1} \sin \underline{\mu} t\right\} \hat{\Xi}^{*} \Lambda^{*} e_{0} \\
=m^{1 / 2} \hat{\Xi}^{-1} \underline{\sim}^{-1} R^{*} \Lambda \Xi \underline{\underline{\lambda}} \sin \left(m^{-1 / 2} \underline{\lambda} t\right) \delta .
\end{gathered}
$$

From these formulas, the integral representations described in Appendix I, and the parity properties of the functions involved follow (III.4).

For (III.3), we note by explicit computation that

$$
\left\|\underline{\mu}^{-1} \hat{\Xi}^{*} e\right\|^{2}=m K_{0}^{-1},
$$

which implies

$$
\begin{aligned}
|C(f, g)| \leqq & \sup _{\substack{v \in \mathbb{R}^{2 N+1} \\
\|v\|=1}} \frac{1}{2 K_{0}} \mid\left(v,\left\{\underline{\mu} h \operatorname{coth}(h \underline{\mu} \beta / 2) \int_{\left(\mathbb{R}^{+}\right)^{2}} e^{-i \zeta(t-s)}\right.\right. \\
& \cdot\left[f^{\prime \prime}(t)-M^{-1} \int_{t}^{\infty} \gamma(t-\tau) f^{\prime}(t)+\rho f(t)\right] \\
& \left.\left.\cdot\left[g^{\prime \prime}(s)-M^{-1} \int_{t}^{\infty} \gamma(s-t) g^{\prime}(t)+\rho g(s)\right] d t d s\right\} v\right) \mid
\end{aligned}
$$

which proves (III.3) after noticing that $\left|\int_{0}^{s} \gamma(t) d t\right| \leqq 2 \sqrt{k / m}$ for each $s \in \mathbb{R}^{+}$.

Now we state the results concerning the thermodynamic limit.

Lemma III.2. For each $f, g \in \mathscr{B}\left(\mathbb{R}^{+}\right)$we have

$$
\begin{aligned}
& \sigma^{L}(f, g)=\lim _{N \rightarrow \infty} \sigma(f, g)=i M^{-2} \int_{\mathbb{R}^{2}} S(f)(t) S(g)^{\prime}(s) \gamma_{\infty}(t-s) d t d s \\
& C^{L}(f, g)=\lim _{N \rightarrow \infty} C(f, g)=\frac{m h}{M^{2}} \int_{-2 \sqrt{k / m}}^{2 \sqrt{k / m}} x \operatorname{coth}(h \beta x / 2) \sqrt{4 k / m-x^{2}} \widehat{S(f)(x) \widehat{S(g)}(x)^{*} d x}
\end{aligned}
$$

where "^" stands for the Fourier transform,

$$
S^{L}: \mathscr{B}\left(\mathbb{R}^{+}\right) \rightarrow \mathscr{B}(\mathbb{R}) \text { is defined by } S^{L}(f)(t)=\left\{\begin{array}{ll}
f(t) & t \geqq 0 \\
\theta_{f}^{L}(t) & t<0
\end{array},\right.
$$

where $\theta_{f}^{L} \in \mathscr{B}\left(\mathbb{R}^{+}\right)$is the unique solution of

$$
\begin{gathered}
\left(\theta_{f}^{L}\right)^{\prime \prime}(t)-M^{-1} \int_{t}^{0} \gamma_{\infty}(t-s)\left(\theta^{L}\right)_{f}^{\prime}(s) d s+\rho \theta_{f}^{L}(t)=M^{-1} \int_{\mathbb{R}^{+}} \gamma_{\infty}(t-s) f^{\prime}(s) d s, \\
\theta_{f}^{L}(0)=f(0) ; \quad\left(\theta^{L}\right)_{f}^{\prime}(0)=f^{\prime}(0)
\end{gathered}
$$

moreover $\gamma_{\infty}(t)=\operatorname{limit}_{N \rightarrow \infty} \gamma(t), \rho=K_{0} / M$.

Proof. It is easy to check that the Fourier transform of $\theta_{f}$ is given by

$$
\begin{aligned}
\hat{\theta}_{f}^{L}(x)= & (2 \pi)^{1 / 2}\left[M^{-1} H_{f}(x)-f^{\prime}(0)-i x f(0)+M^{-1} f^{\prime}(0) \Gamma(x)\right] \\
& \cdot\left[-x^{2}-i M^{-1} x \Gamma(x)+\rho\right]^{-1},
\end{aligned}
$$




$$
\begin{aligned}
H_{f}(x) & =\int_{\mathbb{R}^{-}} e^{-i x t} \int_{\mathbb{R}^{+}} \gamma_{\infty}(t-s) f^{\prime}(s) d s d t, \\
\Gamma(x) & =\int_{\mathbb{R}^{-}} e^{-i x t} \gamma_{\infty}(t) d t .
\end{aligned}
$$

This makes sense because, according to (4.2b) and Appendix I, II, we have

$$
\gamma_{\infty}(t)=\frac{m}{\pi} \int_{-2 \sqrt{k / m}}^{2 \sqrt{k / m}} e^{-i x t} \sqrt{4 K / m-x^{2}} d x
$$

and a direct estimate shows that $\gamma_{\infty} \in L^{1}(\mathbb{R})$. From this it is clear that $\Gamma(\zeta)$ is holomorphic for $\operatorname{Im} \zeta>0$; more precisely we have

$$
\begin{aligned}
& \Gamma(\zeta)=i \frac{4 K \zeta}{\zeta^{2}+i D(\zeta)}, \quad \operatorname{Im} \zeta>0, \\
& \Gamma(x)=\lim _{\varepsilon \rightarrow 0^{+}} \Gamma(x+i \varepsilon), \quad x \in \mathbb{R} .
\end{aligned}
$$

Moreover from $\left[x^{2}\left(\hat{\theta}_{f}^{L}(x)-(x-i)^{-1} f^{\prime}(0)-\left(x^{2}-1\right)^{-1} f(0)\right)\right] \in L^{2}(\mathbb{R})$ we have that $\theta^{L},\left(\theta^{L}\right)^{\prime},\left(\theta^{L}\right)^{\prime \prime} \in L^{2}\left(\mathbb{R}^{-}\right)$and, because $\|f\|_{1} \leqq D\left[\|f\|_{2}+\left\|f^{\prime}\right\|_{2}\right], \theta^{L},\left(\theta^{L}\right)^{\prime} \in L^{1}\left(\mathbb{R}^{-}\right)$ finally using explicitly (III.7) we get $\left(\theta^{L}\right)^{\prime \prime} \in L^{1}\left(\mathbb{R}^{-}\right)$or $\theta^{L} \in \mathscr{B}\left(\mathbb{R}^{-}\right)$. Consequently (III.5) can be verified by direct computation, the same is true for (III.6) as long as we restrict ourselves to $f, g \in C_{0}^{\infty}\left(\mathbb{R}^{+}\right)$. We can nevertheless show that the formula holds for each $f, g \in \mathscr{B}\left(\mathbb{R}^{+}\right)$using (III.3) and the fact that the expression in (III.6) is continuous in $f$ and $g$.

Finally we want to handle the limit $L \rightarrow \infty$, where $m=L^{-1} m^{*}, k=L k^{*}$.

Lemma III.3. $\forall f, g \in \mathscr{B}\left(\mathbb{R}^{+}\right)$, we have

$$
\begin{aligned}
\sigma^{\infty}(f, g) & =\lim _{L \rightarrow \infty} \sigma^{L}(f, g)=2 i \eta M^{-2} \int_{\mathbb{R}} S_{\infty}(f)(x) S_{\infty}(g)^{\prime}(x) d x, \\
C^{\infty}(f, g) & =\lim _{L \rightarrow \infty} C^{L}(f, g) \\
& =\eta M^{-2} \int_{\mathbb{R}} \times h \operatorname{coth}(\beta x / 2) \widehat{S_{\infty}(f)(x) \widehat{S_{\infty}}(g)} *(x) d x,
\end{aligned}
$$

with $\eta=2 \sqrt{k^{*} m^{*}}, S_{\infty}: \mathscr{B}\left(\mathbb{R}^{+}\right) \rightarrow \mathscr{B}(\mathbb{R})$ given by $S_{\infty}(f)(t)=\left\{\begin{array}{ll}f(t) & t \geqq 0 \\ \theta_{f}^{\infty}(t) & t<0\end{array}\right.$, where $\theta_{f}^{\infty} \in \mathscr{B}\left(\mathbb{R}^{-}\right)$is the unique solution of

$$
\left\{\begin{array}{l}
\left(\theta_{f}^{\infty}\right)^{\prime \prime}(t)-\eta M^{-1}\left(\theta_{f}^{\infty}\right)^{\prime}(t)+\rho \theta_{f}^{\infty}(t)=0 \quad t<0 \\
\theta_{f}^{\infty}(0)=f(0), \quad\left(\theta_{f}^{\infty}\right)^{\prime}(0)=f^{\prime}(0)
\end{array} .\right.
$$

Moreover the convergence for $\|f\|^{-1}\|g\|^{-1} \sigma^{L}(f, g),\|f\|^{-1}\|g\|^{-1} C^{L}(f, g)$ is uniform.

Proof. The crucial point is to notice that

where

$$
\gamma^{L}(t)=L \underline{\gamma}(L t)
$$

$$
\underline{\gamma}(t)=\frac{m^{*}}{\pi} \int_{-2 \sqrt{k^{*} / m^{*}}}^{2 \sqrt{k^{*} / m^{*}}} e^{-i x t} \sqrt{4 k^{*} / m^{*}-x^{2}} d x
$$


and that $C^{L}$ can be rewritten as

$$
C^{L}(f, g)=\sqrt{\frac{2}{\pi}} M^{-2} h \int_{\mathbb{R}} x \operatorname{coth}(h \beta x / 2) \widehat{\gamma^{L} * S^{L}}(f)(x) \widehat{S^{L}(g)} *(x) d x .
$$

The result follows then from the fact that $\forall \varepsilon \in \mathbb{R}^{+} \exists \bar{L} \in \mathbb{R}^{+}$:

$$
\begin{array}{ll}
\left\|S^{L}(f)-S^{\infty}(f)\right\|<\varepsilon\|f\| & \forall L>\bar{L}, \\
\left\|\underline{\gamma}^{L} * f-2 \eta f\right\|_{1}<\varepsilon\|f\| & \forall L>\bar{L},
\end{array}
$$

where the second is implied by the estimate $|\gamma(t)|<c_{p} t^{-p} \forall p \in\left(0, \frac{3}{2}\right)$.

Finally notice that if $\operatorname{supp}(f) \subset \mathbb{R}^{+}$, then $S_{\infty}(f)=f$ and we obtain formulas (1.3) (their weak form to be more precise).

Acknowledgements. We thank G. Gallavotti for very useful discussions and suggestions about the problem of the initial condition for the QLE, S. Goldstein for clarifying remarks about the nature of the reduced density matrix in the scaling limit. We also profited from comments by L. Accardi, A. J. Legget, R. J. Rubin, H. Spohn and N. Zanghi.

\section{References}

1. a) Leggett, A. J., Chakravarty, S., Dorsey, A. T., Matthew, P. A., Fisher, A., Garg, A., Zwerger, W.: Rev. Mod. Phys. 59, 1 (1987); b) Chakravarty, S., Leggett, A. J.: Phys. Rev. Lett. 45, 211 (1981); c) Leggett, A. J., Anupam Garg: Phys. Rev. Lett. 54, 857 (1985); d) Grabert, H., Weiss, U.: Phys. Rev. Lett. 54, 1605 (1985); e) Grabert, H., Schramm, P., Ingold, S.-L.: Phys. Rev. Lett. 58, 1285 (1987); f) Fannes, M., Nachtergaele, B., Verbeure, A.: Quantum tunneling in a spin-boson model, Preprint. g) Schnud, A.: JLTP, 49, 609 (1982)

2. For rigorous results see, Dürr, D., Goldstein, S., Lebowitz, J. L.: Commun. Math. Phys. 78, 507 (1981) and references therein

3. Chandrasekhar, S.: Rev. Mod. Phys. 15, 1 (1934)

4. Nelson, Wax eds: Selected papers on Noise and Stochastic process. New York: Dover 1954

5. Ford, G. W., Kac, M., Mazur, P.: J. Math. Phys. 6, 504 (1965); see also, Benguria, K., Kac, M.: Phys. Rev. Lett. 46, 1 (1981)

6. Caldeira, A. O., Legget, A. J.: Physica 121A, 587-616, (1963)

7. Maassen, H.: J. Stat. Phys. 34, 239 (1984)

8. Lewis, J. T., Maasen, H.: In: Quantum probability and applications. Arcadi, L., Frigerio, A., Gorini, V., (eds), Lecture Notes in Mathematics, vol. 1055. Berlin, Heidelberg, New York: Springer 1984

9. Rubin, R. J.: J. Math. Phys. 1, 309 (1960); 2, 373 (1961); Phys. Rev. 131, 964 (1963). See also references there in to earlier work by others on this model

10. Bray, A. J., Moore, M. A.: Phys. Rev. Lett. 49, 1545 (1982)

11. Dürr, D., Naroditsky, V., Zanghi, N.: Lecture Notes in Physics vol., 262, p. 187. Berlin, Heidelberg, New York: Springer 1985

12. Caldeira, A. O., Leggett, A. J.: Phys. Rev. Lett. 46, 211 (1981)

13. Ford, G. W., Kac, M.: J. Stat. Phys. 46, 803 (1987)

14. Schramm, P., Grabert, H.: J. Stat. Phys. (to appear)

15. Feynman, R. P., Vernon, F. L.: Ann. Phys. (NY) 24, 118 (1963)

16. Bratteli, O., Robinson, D. W.: Operator algebras and quantum statistical mechanics I-II. Berlin, Heidelberg, New York: Springer 1979

17. Simon, B.: Functional integration and quantum physics. New York: Academic Press 1979

18. cf Accardi, L., Frigerio, A., Lewis, J. T.: Quantum stochastic processes, Publ. Res. Inst. Math. Sci., Kyoto Univ. 18, 97 (1982)

19. Winnink, M.: In: Statistical Mechanics and field theory. Sen, R. N., Weill, C., (eds). 
20. Reed, M., Simon, B.: Functional analysis. New York: Academic Press 1980

21. Abraham, D., Barouch, E., Gallavotti, G., Martin-Löf., A.: Dynamics of a local perturbation in the $x-y$ model (I-II), Studies in Applied Math. 50, 121-131, (1971) and 52, 211-218, (1972)

22. Lanford III, O. E., Lebowitz, J. L: Time evolution and ergodic properties of harmonic systems. Lecture Notes in Physics vol. 38, Berlin, Heidelberg, New York: Springer 1975 and Lanford III, O. E., Lebowitz, J. L., Lieb, E. H.: J. Stat. Phys. 16, (1977)

Communicated by M. Aizenman

Received February 8, 1988 
